\title{
WEAKLY NONLOCAL NON-EQUILIBRIUM THERMODYNAMICS - VARIATIONAL PRINCIPLES AND SECOND LAW
}

\author{
PÉTER VÁN \\ Dedicated to the 70th birthday of Jüri Engelbrecht.
}

\begin{abstract}
A general, uniform, rigorous and constructive thermodynamic approach to weakly nonlocal non-equilibrium thermodynamics is reviewed. A method is given to construct and restrict the evolution equations of physical theories according to the Second Law of thermodynamics and considering weakly nonlocal constitutive state spaces. The evolution equations of internal variables, the classical irreversible thermodynamics and Korteweg fluids are treated.
\end{abstract}

\section{INTRODUCTION}

Weakly nonlocal, coarse grained, phase field and gradient are attributes of theories from different fields of physics indicating that in contradistinction to the traditional treatments, the governing equations of the theory depend on higher order space derivatives of the state variables. The origin of idea goes back to the square gradient model of van der Waals for phase interfaces [1, where it is extensively applied [2, 3, 4, 5. Later applications go far beyond phase boundaries or thermodynamics. Nowadays weakly nonlocal is a nomination in continuum physics dealing with internal structures [6, 7, 8, 9, 10, 11], coarse grained or phase field appears in statistically motivated thermodynamics $[12,13,14,15,2$, and gradient is frequently used in mechanics in different context [16, 17, 18, 19, 20, 21, 22, 23, 24, 25, 26, 27]. The simplest way to demonstrate the meaning of weakly nonlocal extensions can be exemplified by the Ginzburg-Landau equation which is not only a specific equation in superconductivity, as it was introduced originally by Landau and Khalatnikov 28, but a first weakly nonlocal extension of a homogeneous relaxation equation of an internal variable. The traditional derivation of the Ginzburg-Landau equation is based on a characteristic mixing of variational and thermodynamic considerations. One applies a variational principle for the static part and the functional derivatives are introduced as thermodynamic forces into a relaxation type equation. A clear variational derivation to obtain a first order differential equation is impossible without any further ado (e.g. without introducing new variables to avoid the first order time derivative, which is not a symmetric operator) [29. One can apply these kinds of arguments in continuum theories in general, preserving the doubled theoretical framework separating reversible and irreversible parts of the equations [30, 31, 32. However, there are also other attempts to unify the two parts with different additional hypotheses and to eliminate this inconsistency of the traditional approach [33, 34, 8.

The ultimate aim is to find a unified, general, rigorous and predictive theoretical framework that makes possible to extend the governing equations of physics with higher order gradients of the continuum fields, beyond the traditional terms. The

Date: November 6, 2018. 
method should be uniformly applicable from classical systems in local equilibrium up to relativistic systems beyond local equilibrium; should be general to incorporate most of the mentioned classical examples of weakly nonlocal theories without specific assumptions; should reduce the independent additional assumption to a minimum and should be constructive to give calculational methods for systematic higher order extensions of the constitutive space.

This paper is a general tutorial to the mathematical framework of such a theory. In the first section a general methodology of exploiting the Second Law for weakly nonlocal systems is given. The Second Law is considered as a constrained inequality, where the constraints are the evolution equations of the system and their derivatives, depending on the order of the nonlocality. At the third section evolution equations of internal variables and their different weakly nonlocal extensions are treated. A first order weakly nonlocal theory leads to relaxation type ordinary differential equations, a second order nonlocality leads to Ginzburg-Landau equation and a second order nonlocal theory to dual internal variables unifies the evolution equations of internal variables derived by mechanical methods (by variational principles and dissipation potentials) and by thermodynamics (by heuristic application of the Second Law). At the fourth section we show that classical irreversible thermodynamics can be incorporated naturally in our treatment, a first order weakly nonlocal theory of balance type evolution equations leads to the thermodynamic flux-force relations of classical irreversible thermodynamics with gradients of the intensives as thermodynamic forces. Finally we demonstrate the applicability of the method to one component heat conducting Korteweg fluids, that are first order weakly nonlocal in the energy and in the velocity and second order weakly nonlocal in the density. In that case nontrivial forms of the pressure tensor ensure the compatibility to the Second Law. As a particular example we derive the constitutive functions of the Schrödinger-Madelung fluids. Finally a summary and discussions follow.

\section{SECOND LAW AND WEAKLY NONLOCAL CONSTITUTIVE SPACES}

In this section we shortly summarize some methodological specialities of exploiting the Second Law in weakly nonlocal systems. One can find some further details in [35, 36, where the role of the entropy flux is treated and in [37, where the key idea is introduced, the derivative of the constraints as additional constraint.

There are several interpretations of the Second Law in non-equilibrium thermodynamics (see e.g. 38, 39). If we wanted to ensure that the Second Law will be a consequence of material properties, then we should postulate that the entropy was an increasing function along the solutions of the evolution equations. With this assumption we are compatible with the classical heuristic method that puts the evolution equations in the balance of the entropy, and exploits the consequences of the inequality. In the classical approach a quadratic expression is recognized and then a relation is sought between the introduced thermodynamic fluxes and forces [40, 41. Here the evolution equations are considered as constraints for the inequality of the entropy production [42]. We arrive to the Coleman-Noll procedure recognizing the algebraic part of the problem where the different derivatives are independent. When instead of putting constraints into the evolution equations, the constrained algebraic inequality is solved by multipliers then the calculation is called Liu procedure 43 . The Coleman-Noll and Liu procedures are equivalent in simple systems [44, but the later one preserves the symmetries of the evolution equations and the constraints. Liu procedure is based on a linear algebraic theorem, called Liu's theorem in the thermodynamic literature 45, 46 and an interpretation of the role of entropy inequality. Hauser and Kirchner recognized that Liu's 
theorem is a consequence of a famous statement of optimization theory and linear programming, the so called Farkas's lemma [47. That theorem was proved first by Farkas in 1894 and independently by Minkowski in 1896 [48]. In the Appendix we have formulated and proved the classical Farkas lemma, the affine Farkas lemma and Liu's theorem.

Originally Farkas developed his lemma to formulate correctly the Fourier's principle of mechanics of mass-points, which is the generalization of d'Alembert's principle in case of inequality constraints [49]. The role of Liu's theorem in continuum physics is similar in some sense: we want to give the correct form of the evolution equations taking into account the requirement of the Second Law, that is the entropy inequality. In fact only the material part of the evolution equations - the constitutive functions - is/are restricted. The mathematical formulation introduces a kind of dual point of view, because in Liu procedure the evolution equations - the partial or ordinary differential equations determining the evolution of the system is a condition, a constraint for the entropy inequality. The variables (e.g. fields) in the evolution equation form the basic state space. The constitutive quantities depend on these functions, on the basic state and some of its derivatives. To make the problem algebraically manageable the basic state variables and their derivatives are considered as independent quantities. Some of them can be incorporated into the constitutive state space (or large state space [46]), into the domain of the constitutive functions. The entropy inequality, our objective function, has a special balance form, and determines the process directions, the independent variables of the algebraic problem: those are the derivatives of the constitutive state, that are not included in the constitutive space. The choice of the constitutive state space is crucial and can result in different kind of restricted constitutive functions with Liu procedure.

As we have already mentioned, the weakly nonlocal constitutive spaces contain space derivatives. In this cases some derivatives of the constraints - balances and others - can appear as additional constraints that further restrict the entropy inequality. Whether these additional constraints should be considered or not depends on the order of weak nonlocality, the structure of the constraints and physical considerations. This is the peculiarity of the exploitation of the Second Law for weakly nonlocal systems.

In the following sections we will give several examples to demonstrate the application of the formalism.

\section{THERMODYNAMiC EVOLUTION OF INTERNAL VARIABLES}

In this section we investigate the thermodynamic restrictions on the evolution equation of internal variables in continua at rest. First in a first order weakly nonlocal constitutive state space, then in a second order weakly nonlocal one and finally considering the peculiarities of dual internal variables. Further details of the related calculations and the physical interpretation can be found in [37, 50, 51].

3.1. First order nonlocality - relaxation. Let us investigate the thermodynamic restrictions for the evolution of a classical internal variable field $a(t, \mathbf{r})$, in a continuum at rest related to an inertial observer. There are no constraints or knowledge regarding the form of its evolution equation. Therefore the evolution equation can be given in a general form as

$$
\underline{\partial_{t} a}+\hat{f}=0
$$

with an arbitrary constitutive function $\hat{f}$. Here and in the following the partial time derivatives are denoted by $\partial_{t}$ and the constitutive quantities are denoted by a hat $\left(^{\wedge}\right)$. First we introduce a first order weakly nonlocal constitutive state space spanned 
by the basic fields $a$ and their gradients $\partial_{i} a$, where $i=1,2,3$. The notation with indices with Einstein summation convention is applied by distinguishing covariant and contravariant components (vectors and covectors) by upper and lower indices, e.g. $\partial_{i} J^{i}(=\operatorname{Div} \mathbf{J}=\nabla \cdot \mathbf{J})$ denotes the divergence of the vector field $J^{i}$.

The above evolution equation is not completely arbitrary, it is restricted by the Second Law of thermodynamics. Therefore we assume that there is an entropy balance with a nonnegative production term

$$
\partial_{t} \hat{s}+\partial_{i} \hat{J}^{i} \geq 0
$$

Here the entropy density $\hat{s}$ and the entropy flux $\hat{J}^{i}$ are constitutive quantities, too. Therefore in this case

- the basic state space is spanned by $a$,

- the constitutive state space is spanned by $\left(a, \partial_{i} a\right)$,

- the constitutive functions are $\hat{s}, \hat{J}^{i}$ and $\hat{f}$.

We may develop the derivatives according to the constitutive assumptions as

$$
\partial_{a} \hat{s} \underline{\partial_{t} a}+\partial_{\partial_{i} a} \hat{s} \underline{\partial_{i t} a}+\partial_{a} \hat{J}^{i} \partial_{i} a+\partial_{\partial_{j} a} \hat{J}^{i} \underline{\partial_{i j} a} \geq 0 .
$$

Here the partial derivatives of the constitutive functions are denoted in an abbreviated manner, e.g. $\frac{\partial \hat{s}}{\partial a}=\partial_{a} \hat{s}$. The underlined partial derivatives of the basic field are not in the constitutive space, they are independent algebraic quantities and span the process direction space $\left(\partial_{t} a, \partial_{t i} a, \partial_{i j} a\right)$. Then we can apply the theorem of Liu identifying the underlined partial derivatives in (11) and (3) by $\mathbf{p}$ and their coefficients by $\mathbf{a}$ and $\mathbf{b}$ respectively. Now we introduce a Lagrange-Farkas multiplier $\hat{\lambda}$, which is a constitutive quantity, for the evolution equation (1) and apply Liu procedure to determine the form of the constitutive functions, required by the entropy inequality:

$$
0 \leq\left(\partial_{a} \hat{s}-\hat{\lambda}\right) \underline{\partial_{t} a}+\partial_{\partial_{i} a} \hat{s} \underline{\partial_{i t} a}+\partial_{\partial_{j} a} \hat{J}^{i} \underline{\partial_{i j} a}+\partial_{a} \hat{J}^{i} \partial_{i} a-\hat{\lambda} \hat{f}
$$

Here the existence of the multiplier $\hat{\lambda}$ follows from Liu's theorem and we will exploit that the process directions are independent of the constitutive space in the sense that the values of these derivatives can be different for same values of its coefficients depending e.g. on the initial conditions of (1). Therefore the multipliers of the underlined terms in (4) are zero and give the Liu equations:

$$
\begin{aligned}
\partial_{t} a & : \quad \partial_{a} \hat{s}=\hat{\lambda}, \\
\partial_{i t} a & : \quad \partial_{\partial_{i} a} \hat{s}=0^{i}, \\
\partial_{i j} a & : \quad \partial_{\partial_{j} a} \hat{J}^{i}=0^{i j} .
\end{aligned}
$$

The first equation determines the Lagrange-Farkas multiplier and the last two ones show that both the entropy and the entropy flux is local, independent of the gradient of $a$. This is a complete solution of the system. The residual inequality is

$$
0 \leq \partial_{i} \hat{J}^{i}(a)-\partial_{a} \hat{s}(a) \hat{f}\left(a, \partial_{i} a\right) .
$$

Now we assume that the residual entropy flux is zero $\hat{J}^{i} \equiv 0^{i}$. This is the situation in isotropic materials according to the representation theorem of isotropic vector functions. Therefore the entropy inequality reduces to a flux-force system. If we want to determine the form of the evolution equation then the entropy should be considered as a given function and $\hat{f}$ is an undetermined constitutive quantity. The classical solution of the above inequality is that the constitutive function $\hat{f}$ is proportional to the entropy derivative by a nonnegative constitutive multiplier:

$$
\hat{f}=-\hat{l} \partial_{a} \hat{s}, \quad \hat{l}>0 .
$$


This is a general solution of the inequality if we assume two times differentiability of $\hat{f}$ (due to the mean value theorem of Lagrange, see e.g. [33]). In the practice we rarely exploit this generality and restrict ourselves to the constant $\hat{l}$ case. This remark applies further in (18), (36)-(37) and (53).

Therefore the evolution equation of an internal variable restricted by the Second Law in isotropic materials is a relaxation type differential equation:

$$
\partial_{t} a=\hat{l} \partial_{a} \hat{s}
$$

Now let us summarize the most important steps of the procedure:

- Identification of the basic inequality and the basic constraints and the domain of the corresponding functions (constitutive state space).

- Performing the partial derivations and the identification of the process direction space by the partial derivatives of the basic state space and introducing the additional (derivative) constraints in case of necessity.

- Application of Liu's theorem.

- Solution of the Liu equations and the dissipation inequality.

In this example there was no need to apply additional derivative constraint, but it can be necessary incase of more extended constitutive state spaces as one can see in the next problem

3.2. Second order nonlocality - Ginzburg-Landau equation. In this case we face to a similar problem, we are to determine the thermodynamic restrictions on the general evolution equation (1), but now we assume that there is a second order weakly nonlocal state space spanned by the basic field $a$ and its first and second space derivatives $\partial_{i} a$ and $\partial_{i j} a$.

Therefore in our second example

- the basic state space is spanned by $a$,

- the constitutive state space is spanned by $\left(a, \partial_{i} a, \partial_{i j} a\right)$,

- the constitutive functions are $\hat{s}, \hat{J}^{i}$ and $\hat{f}$.

The corresponding process direction space is spanned by the next derivatives $\left(\partial_{t} a, \partial_{t i} a, \partial_{t i j} a, \partial_{i j k} a\right)$. Let us observe that these are not independent any more, the gradient of (1) is a linear relation on the process direction space. Therefore we should consider

$$
\partial_{t i} a+\partial_{i} \hat{f}=0_{i}
$$

as a further constraint to the entropy inequality (2). We introduce the LagrangeFarkas multipliers $\hat{\lambda}$ for (11) and $\hat{\Lambda}^{i}$ for (11). Let us apply Liu procedure again, but in this case not separating the different steps:

$$
\begin{aligned}
0 \leq & \partial_{t} \hat{s}+\partial_{i} \hat{J}^{i}-\hat{\lambda}\left(\partial_{t} a+\hat{f}\right)-\hat{\Lambda}^{i}\left(\partial_{t i} a+\partial_{i} \hat{f}\right) \\
= & \partial_{a} \hat{s} \partial_{t} a+\partial_{\partial_{i} a} \hat{s} \partial_{i t} a+\partial_{\partial_{i j} a} \hat{s} \partial_{i j t} a+\partial_{a} \hat{J}^{i} \partial_{i} a+\partial_{\partial_{j} a} \hat{J}^{i} \partial_{i j} a+\partial_{\partial_{j k} a} \hat{J}^{i} \partial_{i j k} a \\
& -\hat{\lambda}\left(\partial_{t} a+\hat{f}\right)-\hat{\Lambda}^{i}\left(\partial_{t i} a+\partial_{a} \hat{f} \partial_{i} a+\partial_{\partial_{j} a} \hat{f} \partial_{i j} a+\partial_{\partial_{j k} a} \hat{f} \partial_{i j k} a\right) \\
= & \left(\partial_{a} \hat{s}-\hat{\lambda}\right) \underline{\partial_{t} a}+\left(\partial_{\partial_{i} a} \hat{s}-\hat{\Lambda}^{i}\right) \underline{\partial_{i t} a}+\partial_{\partial_{i j} a} \hat{s} \underline{\partial_{i j t} a+} \\
& \quad\left(\partial_{\partial_{j k} a} \hat{J}^{i}-\hat{\Lambda}^{i} \partial_{\partial_{j k} a} \hat{f}\right) \underline{\partial_{i j k} a}+\partial_{a} \hat{J}^{i} \partial_{i} a+\partial_{\partial_{j} a} \hat{J}^{i} \partial_{i j} a- \\
& \hat{\Lambda}^{i}\left(\partial_{a} \hat{f} \partial_{i} a+\partial_{\partial_{j} a} \hat{f} \partial_{i j} a\right)-\hat{\lambda} \hat{f} .
\end{aligned}
$$

We can see that the degenerations are different than in the previous case. In the following we do not give the detailed expositions of the theorem, but everybody can reconstruct that from the detailed presentation of the calculations. 
The multipliers of the underlined partial derivatives in (12), the process direction space, give the Liu equations:

$$
\begin{aligned}
\partial_{t} a & : \quad \partial_{a} \hat{s}=\hat{\lambda}, \\
\partial_{i t} a & : \quad \partial_{\partial_{i} a} \hat{s}=\hat{\Lambda}^{i}, \\
\partial_{i j t} a & : \quad \partial_{\partial_{i j} a} \hat{s}=0^{i j}, \\
\partial_{i j k} a & : \quad \partial_{\partial_{j k} a} \hat{J}^{i}=\hat{\Lambda}^{i} \partial_{\partial_{j k} a} \hat{f} .
\end{aligned}
$$

The first two equations determine the Lagrange-Farkas multipliers by the entropy derivatives and the third equation shows that the entropy is independent of the second gradient of $a$. As a consequence, the Lagrange-Farkas multiplier $\hat{\Lambda}^{i}$ is independent of that derivative, therefore the last equation can be integrated and gives

$$
\hat{J}^{i}\left(a, \partial_{i} a, \partial_{i j} a\right)=\partial_{\partial_{i} a} \hat{s}\left(a, \partial_{i} a\right) \hat{f}\left(a, \partial_{i} a, \partial_{i j} a\right)+\hat{\mathfrak{J}}^{i}\left(a, \partial_{i} a\right),
$$

where the residual entropy flux, $\hat{\mathfrak{J}}^{i}$, is an arbitrary constitutive function and the variables of the constitutive functions are explicitly written. This is a complete solution of the system of the Liu equations (13)-(16). Therefore the dissipation inequality simplifies to the following form

$$
0 \leq \partial_{i} \hat{\mathfrak{J}}^{i}+\left(\partial_{i}\left(\partial_{\partial_{i} a} \hat{s}\right)-\partial_{a} \hat{s}\right) \hat{f} \text {. }
$$

Assuming that the residual entropy flux is zero $\hat{\mathfrak{J}}^{i} \equiv 0^{i}$, the entropy inequality reduces to a flux-force system. In this case the classical solution of the inequality is

$$
\hat{f}=\hat{l}\left(\partial_{i}\left(\partial_{\partial_{i} a} \hat{s}\right)-\partial_{a} \hat{s}\right), \quad \hat{l}>0 .
$$

Therefore the form of the evolution equation of an internal variable in a second order weakly nonlocal constitutive state space is the Ginzburg-Landau equation:

$$
\partial_{t} a=\hat{l}\left(\partial_{a} \hat{s}-\partial_{i}\left(\partial_{\partial_{i} a} \hat{s}\right)\right) .
$$

We can get the classical form of the equation with a particular form of the entropy density $\hat{s}\left(a, \partial_{i} a\right)=s_{o}(a)-\gamma\left(\partial_{i} a\right)^{2}$, with quadratic dependence on the gradient and with a constant coefficient $\gamma \cdot \gamma>0$ because of the concavity of the entropy. Other nonlocal thermodynamic potentials also can be used in the above derivation, e.g. the free energy, but one should be careful to use a correct thermodynamic structure when coupling to other thermodynamic interactions beyond the one related to the internal variable 13 .

Ginzburg-Landau equation and its variants appear in different fields of physics and are applied to several phenomena. Beyond their original appearance in superconductivity they play an important role in pattern formation and they are the prototypical phase field models [14, 15].

As we have mentioned in the introduction the traditional derivation of the Ginzburg-Landau equation has two main ingredients:

- The static, equilibrium part is derived from a variational principle.

- The dynamic part is added by stability arguments (relaxational form).

The physical content of the two ingredients of the classical derivation is sound and transparent 12. On the other hand, the origin of the variational principle, the coupling of the two parts and the role of the Second Law of thermodynamics is ad-hoc and is not compatible with the general balance and constitutive structure of continuum physics. Here we unified the ingredients in a thermodynamic derivation, and we derived the form of the entropy flux, too. We did not refer to any kind of variational principle, however, the derived static part has a complete EulerLagrange form. The dynamic part contains a first order time derivative, therefore 
one cannot hope to derive it from a variational principle of Hamiltonian type 29. The static part belongs to the zero entropy production, in this sense it is reversible. In our approach we get the "reversible" part as a specific case of the thermodynamic, irreversible thinking.

There are several alternate derivations based on different concepts 33 , 8. Here we have demonstrated, that the physical assumptions to get the Ginzburg-Landau equation are very moderate, the typical Ginzburg-Landau structure is a straightforward consequence of the entropy inequality without any further additional set of concepts like the microforce balance or a variational principle.

3.3. Dual internal variables - Hamiltonian structure. There are two classical approaches to determine the evolution of internal variables.

When the evolution equations of internal variables are constructed exploiting the entropy inequality, using exclusively thermodynamic principles, then the corresponding variables are called internal variables of state 9. This frame has the advantage of operating with familiar thermodynamic concepts (thermodynamic force, entropy), however, no inertial effects are considered. The thermodynamic theory of internal variables has a rich history (see the historical notes in [52]). A first more or less complete thermodynamic theory was suggested by Coleman and Gurtin [53, and the clear presentation of the general ideas of the theory was given by Muschik [54]. Internal variables of state were applied for several phenomena in different areas of physics, biology, and material sciences. A complete description of the thermodynamic theory with plenty of applications based on this concept of internal variables of state can be found in [55].

There is a second method that generates the kinetic relations through the Hamiltonian variational principle and suggests that inertial effects are unavoidable. This approach has a mechanical flavor, and the corresponding variables are called $d y$ namic degrees of freedom. Dissipation is added by dissipation potentials. This theoretical frame has the advantage of operating with familiar mechanical concepts (force, energy). The method was suggested by Maugin [56], and it also has a large number of applications [57, 58]. The clear distinction between these two methods with a number of application areas is given by Maugin and Muschik [52, 59] and Maugin [9].

Here we follow the terminology of Maugin and Muschik [52] with some important extensions. We call internal variables of state those physical field quantities - beyond the classical ones - whose evolution is determined by thermodynamical principles. We call internal degrees of freedom those physical quantities - beyond the classical ones - whose dynamics is determined by mechanical principles.

One of the questions concerning this doubled theoretical frame is related to common application of variational principles and thermodynamics. Basic physical equations of thermodynamical origin do not have variational formulations, at least without any further ado [29. That is well reflected by the appearance of dissipation potentials as separate theoretical entities in variational models dealing with dissipation.

On the other hand, with pure thermodynamical methods - in the internal variables approach - inertial effects are not considered. Therefore, the coupling to simplest mechanical processes seemingly requires some additional assumptions, those are usually new principles of mechanical origin.

On the other hand, with pure thermodynamic methods in the internal variables approach inertial effects are not considered. Therefore, the coupling to simplest mechanical processes seemingly requires introducing some improvements, which are usually new principles of mechanical origin. 
In the following we show that the mechanical structure arises from thermodynamic principles. Our suggestion requires dual internal variables and a particular generalization of the usual postulates of non-equilibrium thermodynamics: we do not require reciprocity relations. With dual internal variables we are able to get inertial effects and to reproduce the evolution of dynamic degrees of freedom. It could be impossible with a single internal variable. This is the price we pay for the generalization. In other words, instead of the doubling of the theoretical structure we suggest the doubling of the number of internal variables.

Let us consider a thermodynamic system where the state space is spanned by two scalar internal variables $a$ and $b$. Then the evolution of these variables is determined by the following differential equations

$$
\begin{aligned}
\partial_{t} a+\hat{f} & =0, \\
\partial_{t} b+\hat{g} & =0 .
\end{aligned}
$$

The functions $\hat{f}$ and $\hat{g}$ are constitutive functions, restricted by the Second Law of thermodynamics. The entropy inequality, the main ingredient of the Second Law can be written in the same form as previously in (2). The domain of the constitutive functions (our constitutive space) is spanned by the state space variables and by their first and second gradients. Therefore in this case

- the basic state space is spanned by $(a, b)$,

- the constitutive state space is spanned by $\left(a, \partial_{i} a, \partial_{i j} a, b, \partial_{i} b, \partial_{i j} b\right)$,

- the constitutive functions are $\hat{s}, \hat{J}^{i}, \hat{f}$ and $\hat{g}$.

This is a weakly nonlocal constitutive space with second order weak nonlocality in both variables. The corresponding process direction space is spanned by the next derivatives $\left(\partial_{t} a, \partial_{t i} a, \partial_{t i j} a, \partial_{i j k} a, \partial_{t} b, \partial_{t i} b, \partial_{t i j} b, \partial_{i j k} b\right)$. The gradients of the evolution equations (21)-(22) are constraints for the entropy inequality in the framework of a second order constitutive state space for both of our variables

$$
\begin{aligned}
& \partial_{t i} a+\partial_{i} \hat{f}=0_{i}, \\
& \partial_{t i} b+\partial_{i} \hat{g}=0_{i} .
\end{aligned}
$$

We introduce the Lagrange-Farkas multipliers $\hat{\lambda}_{a}, \hat{\lambda}_{b}$ for (21)-(22) and $\hat{\Lambda}_{a}^{i}, \hat{\Lambda}_{b}^{i}$ for (23)-(24), respectively. Liu procedure results in the Ginzburg-Landau structure of the previous subsection in a doubled form

$$
\begin{aligned}
& 0 \leq \partial_{t} \hat{s}+\partial_{i} \hat{J}^{i}-\hat{\lambda}_{a}\left(\partial_{t} a+\hat{f}\right)-\hat{\Lambda}_{a}^{i}\left(\partial_{t i} a+\partial_{i} \hat{f}\right)- \\
& \hat{\lambda}_{b}\left(\partial_{t} b+\hat{g}\right)-\hat{\Lambda}_{b}^{i}\left(\partial_{t i} b+\partial_{i} \hat{g}\right) \\
&= \partial_{a} \hat{s} \partial_{t} a+\partial_{\partial_{i} a} \hat{s} \partial_{i t} a+\partial_{\partial_{i j} a} \hat{s} \partial_{i j t} a+\partial_{a} \hat{J}^{i} \partial_{i} a+\partial_{\partial_{j} a} \hat{J}^{i} \partial_{i j} a+\partial_{\partial_{j k} a} \hat{J}^{i} \partial_{i j k} a- \\
& \hat{\lambda}_{a}\left(\partial_{t} a+\hat{f}\right)-\hat{\Lambda}_{a}^{i}\left(\partial_{t i} a+\partial_{a} \hat{f} \partial_{i} a+\partial_{\partial_{j} a} \hat{f} \partial_{i j} a+\partial_{\partial_{j k} a} \hat{f} \partial_{i j k} a+\right. \\
&\left.\partial_{b} \hat{f} \partial_{i} b+\partial_{\partial_{j} b} \hat{f} \partial_{i j} b+\partial_{\partial_{j k} b} \hat{f} \partial_{i j k} b\right) \\
&+\quad \partial_{b} \hat{s} \partial_{t} b+\partial_{\partial_{i} b} \hat{s} \partial_{i t} b+\partial_{\partial_{i j} b} \hat{s} \partial_{i j t} b+\partial_{b} \hat{J}^{i} \partial_{i} b+\partial_{\partial_{j} b} \hat{J}^{i} \partial_{i j} b+\partial_{\partial_{j k} b} \hat{J}^{i} \partial_{i j k} b- \\
& \hat{\lambda}_{b}\left(\partial_{t} b+\hat{g}\right)-\hat{\Lambda}_{b}^{i}\left(\partial_{t i} b+\partial_{b} \hat{g} \partial_{i} b+\partial_{\partial_{j} b} \hat{g} \partial_{i j} b+\partial_{\partial_{j k} b} \hat{g} \partial_{i j k} b+\right. \\
&\left.\partial_{a} \hat{g} \partial_{i} a+\partial_{\partial_{j} a} \hat{g} \partial_{i j} a+\partial_{\partial_{j k} a} \hat{g} \partial_{i j k} a\right) .
\end{aligned}
$$

After some rearrangements one can get 


$$
\begin{aligned}
0 \leq \quad & \left(\partial_{a} \hat{s}-\hat{\lambda}_{a}\right) \partial_{t} a+\left(\partial_{\partial_{i} a} \hat{s}-\hat{\Lambda}_{a}^{i}\right) \partial_{i t} a+\partial_{\partial_{i j} a} \hat{s} \partial_{i j t} a+ \\
& \left(\partial_{\partial_{j k} a} \hat{J}^{i}-\hat{\Lambda}_{a}^{i} \partial_{\partial_{j k} a} \hat{f}-\hat{\Lambda}_{b}^{i} \partial_{\partial_{j k} a} \hat{g}\right) \partial_{i j k} a- \\
& \hat{\lambda}_{a} \hat{f}-\hat{\Lambda}_{a}^{i}\left(\partial_{a} \hat{f} \partial_{i} a+\partial_{\partial_{j} a} \hat{f} \partial_{i j} a+\partial_{b} \hat{f} \partial_{i} b+\partial_{\partial_{j} b} \hat{f} \partial_{i j} b\right)+ \\
& \partial_{a} \hat{J}^{i} \partial_{i} a+\partial_{\partial_{j} a} \hat{J}^{i} \partial_{i j} a \\
+\quad & \left(\partial_{b} \hat{s}-\hat{\lambda}_{b}\right) \partial_{t} b+\left(\partial_{\partial_{i} b} \hat{s}-\hat{\Lambda}_{b}^{i}\right) \partial_{i t} b+\partial_{\partial_{i j} b} \hat{s} \partial_{i j t} b+ \\
& \left(\partial_{\partial_{j k} b} \hat{J}^{i}-\hat{\Lambda}_{a}^{i} \partial_{\partial_{j k} b} \hat{f}-\hat{\Lambda}_{b}^{i} \partial_{\partial_{j k} b} \hat{g}\right) \partial_{i j k} b- \\
& \hat{\lambda}_{b} \hat{g}-\hat{\Lambda}_{b}^{i}\left(\partial_{b} \hat{g} \partial_{i} b+\partial_{\partial_{j} b} \hat{g} \partial_{i j} b+\partial_{a} \hat{g} \partial_{i} a+\partial_{\partial_{j} a} \hat{g} \partial_{i j} a\right)+ \\
& \partial_{b} \hat{J}^{i} \partial_{i} b+\partial_{\partial_{j} b} \hat{J}^{i} \partial_{i j} b .
\end{aligned}
$$

Here the multipliers of the process direction space give the Liu equations:

$$
\begin{aligned}
\partial_{t} a & : \partial_{a} \hat{s}=\hat{\lambda}_{a}, \\
\partial_{i t} a & : \partial_{\partial_{i} a} \hat{s}=\hat{\Lambda}_{a}^{i}, \\
\partial_{t} b & : \partial_{b} \hat{s}=\hat{\lambda}_{b}, \\
\partial_{i t} b & : \partial_{\partial_{i} b} \hat{s}=\hat{\Lambda}_{b}^{i}, \\
\partial_{i j t} a & : \partial_{\partial_{i j} a} \hat{s}=0^{i j}, \\
\partial_{i j t} b & : \partial_{\partial_{i j} b} \hat{s}=0^{i j}, \\
\partial_{i j k} a & : \partial_{\partial_{j k} a} \hat{J}^{i}=\hat{\Lambda}_{a}^{i} \partial_{\partial_{j k} a} \hat{f}+\hat{\Lambda}_{b}^{i} \partial_{\partial_{j k} a} \hat{g}, \\
\partial_{i j k} b & : \partial_{\partial_{j k} b} \hat{J}^{i}=\hat{\Lambda}_{b}^{i} \partial_{\partial_{j k} b} \hat{g}+\hat{\Lambda}_{a}^{i} \partial_{\partial_{j k} b} \hat{f} .
\end{aligned}
$$

The first four equations determine the Lagrange-Farkas multipliers by the entropy derivatives. The fifth and the sixth one show that the entropy is independent of the second gradient of $a$ and $b$. Consequently, the Lagrange-Farkas multipliers $\hat{\Lambda}_{a}^{i}$ and $\hat{\Lambda}_{b}^{i}$ are independent of that derivatives, therefore the last two equations can be integrated and give

$$
\hat{J}^{i}=\partial_{\partial_{i} a} \hat{s} \hat{f}+\partial_{\partial_{i} b} \hat{s} \hat{g}+\hat{\mathfrak{J}}^{i}\left(a, \partial_{i} a, b, \partial_{i} b\right) .
$$

Here the variables of the the residual entropy flux $\hat{\mathfrak{J}}^{i}$, an arbitrary constitutive function, are explicitly written. This is a complete solution of the system of Liu equations (26)-(33). Therefore the dissipation inequality simplifies to the following form

$$
0 \leq \partial_{i} \hat{\mathfrak{J}}^{i}+\left(\partial_{i}\left(\partial_{\partial_{i} a} \hat{s}\right)-\partial_{a} \hat{s}\right) \hat{f}+\left(\partial_{i}\left(\partial_{\partial_{i} b} \hat{s}\right)-\partial_{b} \hat{s}\right) \hat{g} .
$$

Now assuming that the residual entropy flux is zero, $\hat{\mathfrak{J}}^{i} \equiv 0^{i}$, the entropy inequality reduces to a flux-force system of the following form:

$$
\begin{aligned}
& \text { a-force: } \hat{A}=\partial_{i}\left(\partial_{\partial_{i} a} \hat{s}\right)-\partial_{a} \hat{s}, \quad \text { a-flux: } \hat{f} \text {, } \\
& \text { b-force: } \quad \hat{B}=\partial_{i}\left(\partial_{\partial_{i} b} \hat{s}\right)-\partial_{b} \hat{s}, \quad \text { b-flux: } \hat{g} \text {. }
\end{aligned}
$$

The classical solution of the entropy inequality gives a coupling of fluxes and forces as a system of coupled Ginzburg-Landau equations:

$$
\begin{aligned}
\partial_{t} a=\hat{f} & =\hat{l}_{1}\left(\partial_{i}\left(\partial_{\partial_{i} a} \hat{s}\right)-\partial_{a} \hat{s}\right)+\hat{l}_{12}\left(\partial_{i}\left(\partial_{\partial_{i}} \hat{s}\right)-\partial_{b} \hat{s}\right), \\
\partial_{t} a=\hat{g} & =\hat{l}_{21}\left(\partial_{i}\left(\partial_{\partial_{i} a} \hat{s}\right)-\partial_{a} \hat{s}\right)+\hat{l}_{2}\left(\partial_{i}\left(\partial_{\partial_{i}} b \hat{s}\right)-\partial_{b} \hat{s}\right) .
\end{aligned}
$$

The constitutive Onsagerian coefficients $\hat{l}_{1}, \hat{l}_{2}, \hat{l}_{12}, \hat{l}_{21}$ are restricted by the Second Law. For the sake of generality we do not assume any kind of reciprocity here. We 
decouple the symmetric and antisymmetric parts introducing $\hat{l}=\left(\hat{l}_{12}+\hat{l}_{21}\right) / 2$ and $\hat{k}=\left(\hat{l}_{12}-\hat{l}_{21}\right) / 2$. The entropy production is nonnegative if

$$
\hat{l}_{1}>0, \quad \hat{l}_{2}>0 \quad \text { and } \quad \hat{l}_{1} \hat{l}_{2}-\hat{l}^{2} \geq 0
$$

Now the evolution equations (36)-(37) can be written equivalently as

$$
\begin{aligned}
\partial_{t} a & =\hat{k} \hat{B}+\hat{l}_{1} \hat{A}+\hat{l} \hat{B}, \\
\partial_{t} b & =-\hat{k} \hat{A}+\hat{l} \hat{A}+\hat{l}_{2} \hat{B} .
\end{aligned}
$$

3.3.1. Remark on dissipation potentials. We may introduce dissipation potentials for the dissipative part of the equations, if the condition of their existence is satisfied. Dissipation potentials are the children of variational principles, they are artificially added to a set of reversible equations of variational origin to generate some kind of dissipative effects. In our case there is no need of this assumption, our construction gives the most general dissipative system without any further ado. Moreover, here it is clear what belongs to the dissipative part and what belongs to the nondissipative part of the evolution equations. The terms with the symmetric conductivity contribute to the entropy production and the terms from the skew symmetric part do not. On the other hand, there is no need of potential construction, as we are not looking for a variational formulation. Moreover, the symmetry relations are not sufficient for the existence of dissipation potentials in general, as we have emphasized previously. In case of constant coefficients (strict linearity), the dissipation potentials always exist for the dissipative (symmetric) part.

3.3.2. Remark on the reciprocity relations. The reciprocity relations are the main results of the great idea of Lars Onsager connecting fluctuation theory to macroscopic thermodynamics [60, 61, 62, 63, 64]. As it was written by Onsager himself on the validity of his result: "The restriction was stated: on a kinetic model, the thermodynamic variables must be algebraic sums of (a large number of) molecular variables, and must be even functions of those molecular variables which are odd functions of time (like molecular velocities)" 63. The Casimir reciprocity relations are based on microscopic fluctuations, too [64. We do not have such a microscopic background for most of internal variables. E. g. in the case of damage the internal variables are reflecting a structural disorder on a mesoscopic scale. The relation between thermodynamic variables and the microscopic structure is hopelessly complicated. On the other hand, the Onsagerian reciprocity is based on time reversal properties of corresponding physical quantities either at the macro or at the micro level. Looking for the form of evolution equations without a microscopic model, we do not have any information on the time reversal properties of our physical quantities neither at the micro- nor at the macroscopic level. Therefore, we can conclude that lacking the conditions of the Onsagerian or Casimirian reciprocity gives no reasons to assume their validity in the internal variable theory.

Let us observe the correspondence of evolution equations for internal variables with the reciprocity relations by means of a few simple examples.

3.3.3. Example 1: Internal variables. Let us consider materials with diagonal conductivity matrix $\mathbf{L}(\hat{l}=0, \hat{k}=0)$. It is clear that the Onsagerian reciprocity relations are satisfied, and we return to the classical situation with fully uncoupled internal variables:

$$
\begin{aligned}
\partial_{t} a & =\hat{l}_{1} \hat{A}, \\
\partial_{t} b & =\hat{l}_{2} \hat{B} .
\end{aligned}
$$

In this case the evolution equations for dual internal variables $a$ and $b$ are the same as in the case of single internal variable. 
3.3.4. Example 2: Dynamic degrees of freedom. We now assume that all conductivity coefficients are constant, and their values are $l_{1}=l=0, k=1$. This means that $l_{12}=-l_{21}$, i.e., the Casimirian reciprocity relations are satisfied.

For simplicity, we consider a specific decomposition of the entropy density into two parts, which depend on different internal variables

$$
\hat{s}\left(a, \partial_{i} a, b, \partial_{i} b\right)=-K(b)-W\left(a, \partial_{i} a\right) .
$$

The negative signs are introduced taking into account the concavity of the entropy. Then the thermodynamic forces are represented as

$$
\hat{A}=\partial_{a} W-\partial_{i}\left(\partial_{\partial_{i} a} W\right), \quad \hat{B}=d_{b} K=K^{\prime}(b),
$$

and Eqs. (39)-(40) are simplified to

$$
\begin{aligned}
\partial_{t} a & =\hat{B}=K^{\prime}(b) \\
\partial_{t} b & =-\hat{A}+l_{2} \hat{B}=-\partial_{a} W+\partial_{i}\left(\partial_{\partial_{i} a} W\right)+l_{2} K^{\prime}(b) .
\end{aligned}
$$

One may recognize that the obtained system of equation corresponds exactly to a Hamiltonian form with the last term of (42) as added dissipation. Here the entropy density $\hat{s}$ plays the role of a Hamiltonian density, $a$ is a Lagrangian variable and $b$ corresponds to the (slightly generalized) conjugated moment. The transformation into a Lagrangian form is trivial if $K$ is quadratic $K(b)=\frac{b^{2}}{2 m}$, where $m$ is a constant. Then the whole system corresponds to the general structure of dynamic degrees of freedom as one can compare to Eq. (5.14) in [52] with the Lagrangian

$$
\mathcal{L}\left(\partial_{t} a, a, \partial_{i} a\right)=m \frac{\left(\partial_{t} a\right)^{2}}{2}-W\left(a, \partial_{i} a\right), \quad \text { and } \quad D\left(a, \partial_{i} a\right)=\frac{m l_{2}}{2}\left(\partial_{t} a\right)^{2}
$$

as dissipation potential. Moreover, the entropy flux density (34) in case of our special conditions can be written as

$$
J^{i}=-\partial_{\partial_{i} a} s K^{\prime}(b)+\mathfrak{J}_{0}^{i},
$$

and one can infer that natural boundary conditions of the variational principle related to the above Lagrangian correspond to the condition of vanishing entropy flow at the boundary, with $\mathfrak{J}_{0}^{i} \equiv 0^{i}$.

Therefore, the variational structure of internal degrees of freedom is recovered in the pure thermodynamic framework. The thermodynamic structure resulted in several sign restrictions of the coefficients, and the form of the entropy flux is also recovered. The natural boundary conditions correspond to a vanishing extra entropy flux.

3.3.5. Example 3: Diffusive internal variables [65, 66, 67. Now we give an additional example to see clearly the reduction of evolution equations of internal degrees of freedom to evolution equations for internal variables and the extension of the later to the previous one.

We keep the values of conductivity coefficients (i.e., $l_{1}=l=0, k=1$ ), but assume that both $K$ and $W$ are quadratic functions

$$
K(b)=\frac{\beta}{2} b^{2}, \quad W\left(a, \partial_{i} a\right)=\frac{\alpha}{2}\left(\partial_{i} a\right)^{2},
$$

where $\alpha$ and $\beta$ are positive constants according to the concavity requirement.

In this case, the evolution equations (41)-(42) reduce to

$$
\begin{aligned}
\partial_{t} a & =\hat{B}=K^{\prime}(b)=\beta b \\
\partial_{t} b & =-\partial_{a} W+\partial_{i}\left(\partial_{\partial_{i} a} W\right)+l_{2} K^{\prime}(b)=\alpha \partial_{i i} a+l_{2} \beta b .
\end{aligned}
$$


Putting $b$ from Eq. (43) into Eq. (44), we have

$$
\frac{1}{\alpha \beta} \partial_{t t} a-\frac{l_{2}}{\alpha} \partial_{t} a=\partial_{i i} a \text {. }
$$

which is a Cattaneo-Vernotte type hyperbolic equation (telegraph equation) for the internal variable $a$. This can be considered as an extension of a diffusion equation by an inertial term or as and extension of a damped Newtonian equation (without forces) by a diffusion term.

3.3.6. Discussion. In the framework of the thermodynamic theory with dual weakly nonlocal internal variables we are able to recover the evolution equations for internal degrees of freedom.

We have seen that the form of evolution equations depend on the mutual interrelations between the two internal variables. In the special case of internal degrees of freedom, the evolution of one variable is driven by the second one, and vice versa. This can be viewed as a duality between the two internal variables. In the case of pure internal variables of state, this duality is replaced by self-driven evolution for each internal variable. The general case includes all intermediate situations.

It is generally accepted that internal variables are "measurable but not controllable" (see e.g. 68]). Controllability can be achieved by boundary conditions or fields directly acting on the physical quantities. We have seen how natural boundary conditions arise considering nonlocality of the interactions through weakly nonlocal constitutive state spaces.

As we wanted to focus on generic inertial effects, our treatment is simplified from several points of view. Vectorial and tensorial internal variables were not considered and the couplings to traditional continuum fields result in degeneracies and more complicated situations than in our simple examples.

It is important to remark, that skew symmetric couplings are not always related directly to inertial effects and indicate two directions, one into mechanics and one into thermodynamics, where our method can be generalized. Let us mention here the related pioneering works of Verhás, where skew symmetric conductivity equations appear in different inspiring contexts 69, 70.

Finally, let us mention that the idea of constructing a unified theoretical frame for reversible and irreversible dynamics has a long tradition. The corresponding research was not restricted to the case of internal variables and was looking for a classical Hamiltonian or a generalized variational principle that would be valid for both dissipative and nondissipative evolution equations (see, e.g., [71, 72, 73, 74] and the references therein).

\section{Classical Irreversible Thermodynamics}

In this section we investigate the evolution of extensive physical field quantities whose equation of motion is a balance and we will see that for first order weakly nonlocal state spaces the thermodynamic force-flux system is a consequence of the nonnegative entropy production. Treatment of Extended Irreversible Thermodynamics and further details can be found in [36].

A balance type evolution equation for a conserved quantity a can be given in a general form as

$$
\partial_{t} \mathbf{a}+\partial_{i} \hat{\mathbf{j}}^{i}=0
$$

Here $i \in\{1,2,3\}$ denotes the spatial coordinates. The conserved quantity can be a Descartes product of densities of extensives of any tensorial order e.g. $\mathbf{a}=$ $\left(\rho, e, p^{j}, \ldots\right)$, where $\rho$ is the mass density, $e$ is the internal energy density, $p^{j}$ is the momentum density. Our continuum is at rest, $\hat{\mathbf{j}}^{i}$ are the corresponding fluxes, e.g. 
$\mathbf{j}^{i}=\left(\rho v^{i}, \rho e v^{i}+j_{e}^{i}, \rho v^{j} v^{i}+P^{j i}, \ldots\right)$, where the first term is the current of the mass, the second is the convective and conductive current of internal energy and the third is the current of momentum. Therefore with this convenient notation we introduce only those free indices that are important from the point of view of the balance structure of the evolution equation. For the balances of particular real physical quantities there are peculiarities that we do not introduce here. For example for a continuum at rest traditionally there is no mass flux as a consequence of barycentric velocities, the source terms can play an important role (for internal energy, chemical production, etc.). Those peculiarities introduce further constraints and additional terms in the final equations, that we do not consider in this general calculation to show the core structure of classical irreversible thermodynamics. Moreover in this case we restrict ourselves for a continuum at rest. Some consequences of the motion of the continua is considered and investigated at the next section. We assume here a first order weakly nonlocal state space.

Therefore in this case

- the basic state space is spanned by a,

- the constitutive state space is spanned by $\left(\mathbf{a}, \partial_{i} \mathbf{a}\right)$,

- the constitutive functions are $\hat{s}, \hat{J}^{i}$ and $\hat{\mathbf{j}}^{i}$.

The above evolution equation is not completely arbitrary, but restricted by the Second Law of thermodynamics (2). Let us introduce a Lagrange-Farkas multiplier $\lambda$ for the evolution equation (46) and apply Liu procedure to determine the form of the constitutive functions, required by the entropy inequality:

$$
\begin{array}{cc}
0 & \partial_{t} \hat{s}\left(\mathbf{a}, \partial_{i} \mathbf{a}\right)+\partial_{i} \hat{J}^{i}\left(\mathbf{a}, \partial_{i} \mathbf{a}\right)-\hat{\lambda}\left(\partial_{t} \mathbf{a}+\partial_{i} \hat{\mathbf{j}}^{i}\left(\mathbf{a}, \partial_{i} \mathbf{a}\right)\right) \\
= & \partial_{\mathbf{a}} \hat{s} \partial_{t} \mathbf{a}+\partial_{\partial_{i} \mathbf{a}} \hat{s} \partial_{i t} \mathbf{a}+\partial_{\mathbf{a}} \hat{J}^{i} \partial_{i} \mathbf{a}+\partial_{\partial_{j} \mathbf{a}} \hat{J}^{i} \partial_{i j} \mathbf{a} \\
& -\hat{\lambda}\left(\partial_{t} \mathbf{a}+\partial_{\mathbf{a}} \hat{\mathbf{j}}^{i} \partial_{i} \mathbf{a}+\partial_{\partial_{j}} \hat{\mathbf{j}}^{i} \partial_{i j} \mathbf{a}\right) \\
= & \left(\partial_{\mathbf{a}} \hat{s}-\hat{\lambda}\right) \underline{\partial_{t} \mathbf{a}}+\partial_{\partial_{i} \mathbf{a}} \hat{s} \underline{\partial_{i t} \mathbf{a}}+\left(\partial_{\mathbf{a}} \hat{J}^{i}-\hat{\lambda} \partial_{\mathbf{a}} \hat{\mathbf{j}}\right) \partial_{i} \mathbf{a} \\
& +\left(\partial_{\partial_{j} \mathbf{a}} \hat{J}^{i}-\hat{\lambda} \partial_{\partial_{j}} \hat{\mathbf{j}}^{i}\right) \underline{\partial_{i j} \mathbf{a}}
\end{array}
$$

The underlined partial derivatives of the basic field are not in the constitutive space, they are independent algebraic quantities and span the process direction space. The multipliers of those terms are zero according to Liu's theorem and give the Liu equations:

$$
\begin{aligned}
& \partial_{t} \mathbf{a}: \partial_{\mathbf{a}} \hat{s}=\hat{\lambda}, \\
& \partial_{i t} \mathbf{a}: \partial_{\partial_{i} \mathbf{a}} \hat{s}=0^{i}, \\
& \partial_{i j} \mathbf{a} \quad: \quad \partial_{\partial_{j} \mathbf{a}} \hat{J}^{i}=\hat{\lambda} \partial_{\partial_{j}} \mathbf{a} \hat{\mathbf{j}}^{i} .
\end{aligned}
$$

The first equation determines the Lagrange-Farkas multiplier and the second one shows that the entropy should be local, independent of the gradient of $\mathbf{a}$. Therefore one can solve the third equation as

$$
\hat{J}^{i}\left(\mathbf{a}, \partial_{i} \mathbf{a}\right)=\partial_{\mathbf{a}} s(\mathbf{a}) \cdot \hat{\mathbf{j}}^{i}\left(\mathbf{a}, \partial_{i} \mathbf{a}\right)+\hat{\mathfrak{J}}^{i}(\mathbf{a}),
$$

where we have denoted the variables of the constitutive functions and we have introduced the local residual entropy flux $\hat{\mathfrak{J}}^{i}$. Several authors suggest this kind of additive supplement to the classical entropy flux (e.g. the $\mathbf{K}$ vector of Müller [75]). This is a complete solution of the system (48)-(50). The dissipation inequality is

$$
0 \leq \partial_{i} \hat{\mathfrak{J}}^{i}+\hat{\mathbf{j}}^{i} \partial_{i}\left(\partial_{\mathbf{a}} \hat{s}\right) .
$$

Assuming that the residual entropy flux is zero $\hat{\mathfrak{J}}^{i} \equiv 0^{i}$, the entropy inequality reduces to the usual flux-force system of Classical Irreversible Thermodynamics, 
where the thermodynamic forces are the gradients of the intensives and the thermodynamic fluxes are identical to the the fluxes of the extensives from the balances.

$$
\text { Flux: } \hat{\mathbf{j}}^{i}, \quad \text { Force: } \partial_{i}\left(\partial_{\mathbf{a}} \hat{s}\right) \text {. }
$$

The classical solution of the above inequality is that the fluxes are proportional to the forces:

$$
\hat{\mathbf{j}}^{i}=\hat{\mathbf{L}}^{i k} \partial_{k}\left(\partial_{\mathbf{a}} \hat{s}\right),
$$

where $\hat{\mathbf{L}}^{i k}$ is symmetric and positive definite. Therefore balances of extensives are reduced to transport equations of the form:

$$
\partial_{t} \mathbf{a}+\partial_{i}\left(\mathbf{L}^{i j} \partial_{j}\left(\partial_{\mathbf{a}} \hat{s}\right)\right)=0 .
$$

We have seen that in our calculations the classical form of the entropy production (the first term in the inequality above) and the classical form of the entropy flux were consequences of the Second Law, with the assumption of first order nonlocality. A rigorous treatment showed that the seemingly intuitive steps of irreversible thermodynamic modelling are well supported and explained by Liu procedure. We can see that the second part of the usual phenomenological formulation of the local equilibrium hypothesis [72] is a consequence of the assumption of first order nonlocality: the state functions are those in equilibrium due to the local entropy.

\section{ONE COMPONENT FLUIDS - SECOND ORDER NONLOCAL IN THE DENSITY}

Up to know we have investigated evolution equations of internal variables and the general structure of Classical Irreversible Thermodynamics in a somewhat abstract manner. In this section we analyze a more particular and less abstract example where the physical meaning of the variables in the basic state space is well known. Our example is a one component heat conducting fluid where the constitutive state space for the energy and the velocity fields are first order, but second order in the density. We have seen that in case of first order weakly nonlocal state spaces our method gives the well known classical structure with a somewhat simplified manner, where the number of independent assumptions are reduced. E.g. the form of the entropy flux is calculated and not postulated, as we have demonstrated in the previous section. However, the second order nonlocality in the density variables leads to a surprising result and gives the viable family of Korteweg fluids, those that are compatible with the Second Law. One can find more details in [76, 77. and with alternate methods in [78, 11.

5.1. Fluid mechanics in general. The basic state space of one-component fluid mechanics is spanned by the mass density $\rho$, the velocity $v^{i}$ and the energy density $e$ of the fluid. Hydrodynamics is based on the balance of mass, energy and momentum [71. In classical fluid mechanics the constitutive space, the domain of the constitutive functions, is spanned by the basic state space $\left(\rho, v^{i}, e\right)$ and the gradient of the velocity $\partial_{i} v^{j}$ and the temperature $\partial_{i} T$. The pressure/stress tensor and the flux of the internal energy are the constitutive quantities in the theory.

The balance of mass can be written as

$$
\partial_{t} \rho+\partial_{i}\left(\rho v^{i}\right)=0 .
$$

There is only a convective flux for mass. The balance of momentum, i.e. the Cauchy equation, is

$$
\partial_{t}\left(\rho v^{i}\right)+\partial_{j}\left(\hat{P}^{i j}+\rho v^{i} v^{j}\right)=0^{i},
$$

where $\rho v^{i}$ is the momentum density and $\hat{P}^{i j}$ is the pressure tensor, the conductive flux of the momentum. 
The balance of energy is given as

$$
\partial_{t} e+\partial_{i}\left(\hat{q}^{i}+e v^{i}\right)=0
$$

Here $e$ is the total energy density and $\hat{q}^{i}$ is the conductive flux of the energy. The Second Law requires that the production of the entropy is nonnegative in insulated and source-free systems, therefore source terms are not considered in the balances. Hence in our case there is no mass production, the external forces are absent and the energy is conserved. We do not need the concept of the internal energy yet, first we analyse the consequences of the Second Law with the balance of the total energy. As we are dealing with a fluid, it is convenient to separate the conductive and convective entropy fluxes. Hence (2) will be written as

$$
\partial_{t} \hat{s}+\partial_{i}\left(\hat{J}^{i}+\hat{s} v^{i}\right) \geq 0
$$

Therefore in this case

- the basic state space is spanned by $\left(\rho, v^{i}, e\right)$,

- the constitutive state space is spanned by $\left(\rho, \partial_{i} \rho, \partial_{i j} \rho, v^{i}, \partial_{i} v^{j}, e, \partial_{i} e\right)$,

- the constitutive functions are $\hat{s}, \hat{J}^{i}, \hat{q}^{i}$ and $\hat{P}^{i j}$.

It is somewhat convenient to introduce the relative velocity $v^{i}$ as basic state variable (instead of the momentum) and the gradient of the energy as constitutive state variable (instead of the temperature gradient). As we have a second order weakly nonlocal extension in the mass density we need the gradient of (55) as a further constraint in the entropy inequality

$$
\partial_{i t} \rho+\partial_{i j}\left(\rho v^{j}\right)=0_{i}
$$

We introduce the Lagrange-Farkas multipliers $\hat{\lambda}, \hat{\Lambda}^{i}, \hat{\Gamma}_{i}, \hat{\gamma}$ for the balances (55), (59), (56) and (57) respectively.

Now we apply Liu procedure, with the method of Lagrange-Farkas multipliers as in the previous sections

$$
\begin{aligned}
0 \leq & \partial_{t} \hat{s}+\partial_{i} \hat{J}^{i}+\partial_{i}\left(\hat{s} v^{i}\right)-\hat{\lambda}\left(\partial_{t} \rho+\partial_{i}\left(\rho v^{i}\right)\right)-\hat{\Lambda}^{i}\left(\partial_{i t} \rho+\partial_{i j}\left(\rho v^{j}\right)\right)- \\
& \hat{\Gamma}_{i}\left(\partial_{t}\left(\rho v^{i}\right)+\partial_{j}\left(\hat{P}^{i j}+\rho v^{i} v^{j}\right)\right)-\hat{\gamma}\left(\partial_{t} e+\partial_{i}\left(\hat{q}^{i}+e v^{i}\right)\right) .
\end{aligned}
$$

Developing the partial derivatives of the constitutive functions gives:

$$
\begin{gathered}
\partial_{\rho} \hat{s} \partial_{t} \rho+\partial_{\partial_{i} \rho} \hat{s} \partial_{t i} \rho+\partial_{\partial_{i j} \rho} \hat{s} \partial_{t i j} \rho+\partial_{v^{i}} \hat{s} \partial_{t} v^{i}+\partial_{\partial_{i} v^{j}} \hat{s} \partial_{t j} v^{i}+\partial_{e} \hat{s} \partial_{t} e+ \\
\partial_{\partial_{i} e} \hat{s} \partial_{t i} e+\partial_{\rho} \hat{J}^{i} \partial_{i} \rho+\partial_{\partial_{j} \rho} \hat{J}^{i} \partial_{i j} \rho+\partial_{\partial_{j k} \rho} \hat{J}^{i} \partial_{i j k} \rho+\partial_{v^{j}} \hat{J}^{i} \partial_{i} v^{j}+\partial_{\partial_{k} v^{j}} \hat{J}^{i} \partial_{j k} v^{i}+ \\
\partial_{e} \hat{J}^{i} \partial_{i} e+\partial_{\partial_{j} e} \hat{J}^{i} \partial_{i j} e+ \\
\partial_{i}\left(\hat{s} v^{i}\right)-\hat{\lambda}\left(\partial_{t} \rho+\rho \partial_{i} v^{i}+v^{i} \partial_{i} \rho\right)- \\
\hat{\Lambda}^{i}\left(\partial_{i t} \rho+\rho \partial_{i j} v^{j}+v^{j} \partial_{i j} \rho+\partial_{i} \rho \partial_{j} v^{j}+\partial_{j} \rho \partial_{i} v^{j}\right)- \\
\hat{\Gamma}_{i}\left(\rho \partial_{t} v^{i}+v^{i} \partial_{t} \rho+\rho v^{i} \partial_{j} v^{j}+\rho v^{j} \partial_{j} v^{i}+v^{j} v^{i} \partial_{j} \rho+\partial_{\rho} \hat{P}^{i j} \partial_{j} \rho+\partial_{\partial_{k} \rho} \hat{P}^{i j} \partial_{j k} \rho+\right. \\
\left.\partial_{\partial_{k l} \rho} \hat{P}^{i j} \partial_{j k l} \rho+\partial_{v^{k}} \hat{P}^{i j} \partial_{j} v^{k}+\partial_{\partial_{l} v^{k}} \hat{P}^{i j} \partial_{j l} v^{k}+\partial_{e} \hat{P}^{i j} \partial_{j} e+\partial_{\partial_{k} e} \hat{P}^{i j} \partial_{j k} e\right)- \\
\hat{\gamma}\left(\partial_{t} e+e \partial_{i} v^{i}+v^{i} \partial_{i} e+\partial_{\rho} \hat{q}^{i} \partial_{i} \rho+\partial_{\partial_{j} \rho} \hat{q}^{i} \partial_{j i} \rho+\right. \\
\left.\partial_{\partial_{j k} \rho} \hat{q}^{i} \partial_{i j k} \rho+\partial_{v^{j}} \hat{q}^{i} \partial_{i} v^{j}+\partial_{\partial_{k} v^{j}} \hat{q}^{i} \partial_{i k} v^{j}+\partial_{e} \hat{q}^{i} \partial_{i} e+\partial_{\partial_{j}} \hat{q}^{i} \partial_{j i} e\right) \geq 0 .
\end{gathered}
$$


A little rearrangement of the terms results in

$$
\begin{gathered}
\left(\partial_{\rho} \hat{s}-\hat{\lambda}-\hat{\Gamma}_{i} v^{i}\right) \partial_{t} \rho+\left(\partial_{\partial_{i} \rho} \hat{s}-\hat{\Lambda}^{i}\right) \partial_{t i} \rho+\partial_{\partial_{i j} \rho} \hat{s} \partial_{t i j} \rho+\left(\partial_{v^{i}} \hat{s}-\rho \hat{\Gamma}_{i}\right) \partial_{t} v^{i}+ \\
\partial_{\partial_{i} v^{j}} \hat{s} \partial_{t j} v^{i}+\left(\partial_{e} \hat{s}-\hat{\gamma}\right) \partial_{t} e+\partial_{\partial_{i} e} \hat{s} \partial_{t i} e+ \\
\left(\partial_{\partial_{j k} \rho} \hat{J}^{i}-\hat{\Gamma}_{l} \partial_{\partial_{j k} \rho} \hat{P}^{l i}-\partial_{\partial_{j k} \rho} \hat{q}^{i}\right) \partial_{i j k} \rho+ \\
\left(\partial_{\partial_{k} v^{j}} \hat{J}^{i}-\hat{\Gamma}_{l} \partial_{\partial_{k} v^{i}} \hat{P}^{l j}-\hat{\gamma} \partial_{\partial_{k} v^{i}} \hat{q}^{j} \partial_{i k} v^{j}\right) \partial_{j k} v^{i}-\hat{\Lambda}^{i} \rho \partial_{i j} v^{j}+ \\
\left(\partial_{\partial_{j} e} \hat{J}^{i}-\hat{\Gamma}_{l} \partial_{\partial_{i} e} \hat{P}^{l j}+\hat{\gamma} \partial_{\partial_{j} e} \hat{q}^{i}\right) \partial_{i j} e+ \\
\partial_{\rho} \hat{J}^{i} \partial_{i} \rho+\partial_{\partial_{j} \rho} \hat{J}^{i} \partial_{i j} \rho+\partial_{v^{j}} \hat{J}^{i} \partial_{i} v^{j}+\partial_{e} \hat{J}^{i} \partial_{i} e+ \\
\partial_{i}\left(\hat{s} v^{i}\right)-\hat{\lambda}\left(\rho \partial_{i} v^{i}+v^{i} \partial_{i} \rho\right)- \\
\hat{\Lambda}^{i}\left(\rho \partial_{i j} v^{j}+v^{j} \partial_{i j} \rho+\partial_{i} \rho \partial_{j} v^{j}+\partial_{j} \rho \partial_{i} v^{j}\right)- \\
\hat{\Gamma}_{i}\left(\rho v^{i} \partial_{j} v^{j}+\rho v^{j} \partial_{j} v^{i}+v^{j} v^{i} \partial_{j} \rho+\partial_{\rho} \hat{P}^{i j} \partial_{j} \rho+\partial_{\partial_{k} \rho} \hat{P}^{i j} \partial_{j k} \rho+\right. \\
\left.\partial_{v^{k}} \hat{P}^{i j} \partial_{j} v^{k}+\partial_{e} \hat{P}^{i j} \partial_{j} e\right)- \\
\hat{\gamma}\left(e \partial_{i} v^{i}+v^{i} \partial_{i} e+\partial_{\rho} \hat{q}^{i} \partial_{i} \rho+\partial_{\partial_{j} \rho} \hat{q}^{i} \partial_{j i} \rho+\partial_{v^{j}} \hat{q}^{i} \partial_{i} v^{j}+\partial_{e} \hat{q}^{i} \partial_{i} e\right) \geq 0 .
\end{gathered}
$$

Then the Liu-equations follow as the multipliers of the members of the process direction space, the derivatives that are out of the constitutive space

$$
\begin{aligned}
\partial_{t} \rho & : \partial_{\rho} \hat{s}-\hat{\lambda}-\hat{\Gamma}_{i} v^{i}=0 \\
\partial_{t i} \rho & : \partial_{\partial_{i} \rho} \hat{s}-\hat{\Lambda}^{i}=0^{i} \\
\partial_{t i j} \rho & : \quad \partial_{\partial_{i j} \rho} \hat{s}=0^{i j} \\
\partial_{t} v^{i} & : \quad \partial_{v^{i}} \hat{s}-\rho \hat{\Gamma}_{i}=0_{i}, \\
\partial_{t j} v^{i} & : \quad \partial_{\partial_{j} v^{i}} \hat{s}=0_{i}^{j}, \\
\partial_{t} e & : \quad \partial_{e} \hat{s}-\hat{\gamma}=0, \\
\partial_{t i} e & : \quad \partial_{\partial_{i} e} \hat{s}=0^{i}, \\
\partial_{i j k} \rho & : \quad \partial_{\partial_{k j} \rho} \hat{J}^{i}=\hat{\Gamma}_{l} \partial_{\partial_{k j} \rho} \hat{P}^{l i}+\hat{\gamma} \partial_{\partial_{k j} \rho} \hat{q}^{i}, \\
\partial_{j k} v^{i} & : \quad \partial_{\partial_{k} v^{i}} \hat{J}^{j}=\hat{\Gamma}_{l} \partial_{\partial_{k} v^{i}} \hat{P}^{l j}+\hat{\gamma} \partial_{\partial_{k} v^{i}} \hat{q}_{j}+\frac{\rho}{2} \hat{\Lambda}^{l}\left(\delta_{l}^{j} \delta_{i}^{k}-\delta_{l}^{k} \delta_{i}^{j}\right), \\
\partial_{i j} e & : \quad \partial_{\partial_{j} e} \hat{J}^{i}=\hat{\Gamma}_{l} \partial_{\partial_{i} e} \hat{P}^{l j}+\hat{\gamma} \partial_{\partial_{j} e} \hat{q}^{i} .
\end{aligned}
$$

As a consequence of (63), (65) and (67), the entropy density does not depend on $\partial_{i j} \rho, \partial_{j} v^{i}$ and $\partial_{i} e$. (61), (62), (64) and (66) give the Lagrange-Farkas multipliers in terms of the entropy derivatives. Therefore, from a thermodynamic point of view, the Lagrange-Farkas multipliers are the normal and generalized intensive variables [79]. Now, one can give a solution of (68)-(70) as

(71) $\hat{J}^{i}=\partial_{e} \hat{s} \hat{q}^{i}+\frac{\rho}{2}\left(\partial_{\partial_{i} \rho} \hat{s} \partial_{j} v^{j}+\partial_{\partial_{j} \rho} \hat{s} \partial_{j} v^{i}\right)+\frac{1}{\rho} \partial_{v^{j}} \hat{s} \hat{P}^{j i}+\hat{\mathfrak{J}}^{i}\left(\rho, \partial_{i} \rho, v^{i}, e\right)$,

where the residual entropy flux $\hat{\mathfrak{J}}^{i}$ is an arbitrary function. Thus Liu's equations can be solved and yield the Lagrange-Farkas multipliers as well as restrictions for the entropy and the entropy flux. Applying these solutions of the Liu equations, the dissipation inequality can be simplified to the following form

$$
\begin{aligned}
0 \leq \sigma_{s}= & \partial_{i} \hat{\mathfrak{J}}^{i}+\hat{q}^{i} \partial_{i}\left(\partial_{e}(\rho \mathfrak{s})\right)+\hat{P}^{i j} \partial_{i}\left(\partial_{\left.v^{j} \mathfrak{s}\right)}+\right. \\
& \partial_{j} v^{j}\left(\hat{s}+e \partial_{e} \hat{s}-\rho \partial_{\rho} \hat{s}+\frac{\rho^{2}}{2} \partial_{i}\left(\partial_{\partial_{i} \rho \mathfrak{s}}\right)\right)+\partial_{j} v^{i}\left(\frac{\rho^{2}}{2} \partial_{i}\left(\partial_{\partial_{j} \rho} \mathfrak{s}\right)\right) .
\end{aligned}
$$


Here we have introduced the specific entropy as $\mathfrak{s}:=\hat{s} / \rho$. It is worth to give this inequality, the entropy production in a more traditional form, without indices, too

$$
\begin{aligned}
& \nabla \cdot \mathbf{J}_{0}+\mathbf{q} \cdot \nabla \partial_{e}(\rho \mathfrak{s})+ \\
& \quad \nabla \partial_{\mathbf{v}} \mathfrak{s}: \mathbf{P}+\left[\frac{\rho^{2}}{2}\left(\nabla \cdot \partial_{\nabla \rho \mathfrak{s}} \mathbf{I}+\nabla \partial_{\nabla \rho \mathfrak{s}}\right)+\left(\hat{s}+e \partial_{e} \hat{s}-\rho \partial_{\rho} \hat{s}\right) \mathbf{I}\right]: \nabla \mathbf{v} \geq 0 .
\end{aligned}
$$

Here $\mathbf{I}$ denotes the second order unit tensor $\delta_{i j}, \nabla$ is the gradient, $\nabla \cdot$ is the divergence of the corresponding field quantity. To get the traditional form of the equation we should introduce the internal energy of the fluid as the difference of the total and kinetic energies and assume that the entropy function, does not depend on the total and kinetic energies independently, but only on the internal energy $\hat{s}=\hat{s}\left(\rho, \partial_{i} \rho, e-\rho v^{2} / 2\right)$. Moreover, we want to define the entropy as an extensive quantity, therefore we require that the specific entropy depends on the specific internal energy $\epsilon$ :

$$
\hat{s}\left(\rho, \partial_{i} \rho, v^{i}, e\right)=\rho \mathfrak{s}\left(\rho, \frac{e}{\rho}-\frac{v^{2}}{2}, \partial_{i} \rho\right) .
$$

From this form of the entropy function we get the Gibbs relation

$$
d \epsilon=T d \mathfrak{s}+\frac{p}{\rho^{2}} d \rho-A^{i} d \partial_{i} \rho .
$$

where $\epsilon=\frac{e}{\rho}-\frac{v^{2}}{2}$ is the specific internal energy. The temperature and pressure are defined by the customary partial derivatives of the entropy. The temperature can be connected both to the derivative by the total energy and the internal energy, because the corresponding derivatives are equal. $A^{i}$ is defined as the partial derivative of the entropy by the density gradient, like the traditional intensives. :

$$
\partial_{e}(\rho \mathfrak{s})=\frac{1}{T}, \quad \partial_{v^{j}} \mathfrak{s}=-\frac{v^{j}}{T}, \quad \partial_{\rho} \mathfrak{s}=-\frac{p}{T \rho^{2}}, \quad \partial_{\partial_{i} \rho} \mathfrak{s}=\frac{A^{i}}{T} .
$$

With these quantities we can write the dissipation inequality and the entropy flux as

$$
\begin{aligned}
0 \leq \sigma_{s}=\partial_{i} \hat{\mathfrak{J}}^{i}+ & \left(\hat{q}^{i}-v_{j} \hat{P}^{i j}\right) \partial_{i} \frac{1}{T}- \\
& \frac{1}{T}\left(\hat{P}^{i j}-\left(p+\frac{T \rho^{2}}{2} \partial_{k}\left(\partial_{\partial_{k} \rho} \mathfrak{s}\right)\right) \delta_{j}^{i}-\frac{T \rho^{2}}{2} \partial_{j}\left(\partial_{\partial_{i} \rho \mathfrak{s}}\right)\right) \partial_{i} v^{j} \\
\hat{J}^{i}= & \left(\hat{q}^{i}-v_{j} \hat{P}^{j i}\right) \frac{1}{T}+\frac{\rho}{2}\left(\partial_{\partial_{i} \rho} \hat{s} \partial_{j} v^{j}+\partial_{\partial_{k} \rho} \hat{s} \partial_{k} v^{i}\right)+\hat{\mathfrak{J}}^{i} .
\end{aligned}
$$

We can see that a flux of the internal energy is introduced as in case of the traditional, first order weakly nonlocal theories. However, the appearance of temperature inside the expression of the viscous pressure indicates the possibility of alternate, better definitions.

Now we change the notation to a coordinate invariant one, introducing the nabla operator for the space derivatives as it is customary in fluid mechanics. In this case e.g. $\partial_{i} v^{i}=\nabla \cdot \mathbf{v}$ and $\partial_{i} v^{j}=\nabla \mathbf{v}$. In the pure mechanical, reversible case our thermodynamic force for mechanical interactions is zero. Therefore we introduce the nonlocal reversible pressure as

$$
\hat{\mathbf{P}}^{r}=\frac{T \rho^{2}}{2}\left[\left(\nabla \cdot \partial_{\nabla \rho \mathfrak{s}}-2 \partial_{\rho \mathfrak{s}}\right) \mathbf{I}+\nabla \partial_{\nabla \rho \mathfrak{s}}\right] .
$$

If the pressure is equal to the reversible pressure, there is no dissipation, the theory is reversible (conservative). In case of a local entropy (independent of the 
gradient of the density) then we obtain

$$
\hat{\mathbf{P}}_{\text {Euler }}^{\mathrm{r}}(\rho):=-T \rho^{2} \partial_{\rho} \mathfrak{s}(\rho) \mathbf{I},
$$

therefore, the corresponding equations are of the ideal Euler fluid, where $p(\rho)=$ $-T \rho^{2} \partial_{\rho} \mathfrak{s}(\rho)$ is the scalar pressure function. Introducing the viscous pressure $\mathbf{P}^{\mathbf{v}}$ as usual, we can solve the dissipation inequality and give the corresponding Onsagerian conductivity equation as

$$
\hat{\mathbf{P}}^{\mathrm{v}}:=\hat{\mathbf{P}}-\hat{\mathbf{P}}^{\mathrm{r}}=\mathbf{L}_{O N S} \cdot \nabla \mathbf{v} .
$$

Here $\mathbf{L}_{O N S}$ is a nonnegative constitutive function. Let us recognize that if $\mathfrak{s}$ is independent of the gradient of the density, $\mathbf{L}_{O N S}$ is constant, and $\hat{\mathbf{P}}^{\mathrm{v}}$ is an isotropic function of only $\nabla \mathbf{v}$, then we obtain the traditional Navier-Stokes fluid (see e.g. [71]).

One can prove easily that the reversible part of the pressure is potentializable, i.e., there is a scalar valued function $U$ such that

$$
\nabla \cdot \hat{\mathbf{P}}^{\mathrm{r}}=\rho \nabla \hat{U} .
$$

$\hat{U}$ can be calculated from the entropy function as

$$
\hat{U}=\nabla \cdot\left(\rho \partial_{\nabla \rho \mathfrak{s}}\right)-\partial_{\rho}(\rho \mathfrak{s}) .
$$

Therefore in case of reversible fluids the momentum balance can be written alternatively as

$$
\rho \dot{\mathbf{v}}+\nabla \cdot \hat{\mathbf{P}}^{r}=\mathbf{0} \quad \Longleftrightarrow \quad \dot{\mathbf{v}}+\nabla \hat{U}=\mathbf{0} .
$$

Let us give an interesting particular example of a weakly nonlocal fluid.

5.2. Schrödinger-Madelung fluid. Here the entropy is defined as

$$
s_{\mathrm{SchM}}(\rho, \nabla \rho, \mathbf{v})=-\frac{\nu}{2}\left(\frac{\nabla \rho}{2 \rho}\right)^{2}-\frac{\mathbf{v}^{2}}{2}=-\frac{\nu}{8}(\nabla \ln \rho)^{2}-\frac{\mathbf{v}^{2}}{2},
$$

where $\nu$ is a constant scalar. The corresponding reversible pressure is

$$
\mathbf{P}^{\mathrm{r}}=-\frac{\nu}{8}\left(\Delta \rho \mathbf{I}+\nabla^{2} \rho-\frac{2 \nabla \rho \circ \nabla \rho}{\rho}\right)
$$

where $\circ$ denotes the tensorial/dyadic product, as mentioned before. The potential is

$$
U_{\mathrm{SchM}}=-\frac{\nu}{4 \rho}\left(\Delta \rho-\frac{(\nabla \rho)^{2}}{2 \rho}\right)=-\frac{\nu}{2} \frac{\Delta R}{R},
$$

where we introduced $R=\sqrt{\rho}$ to show that (84) is the quantum potential in the de Broglie-Bohm version of quantum mechanics (if $\nu=\hbar^{2} / m^{2}$ ) [80, 81].

The entropy flux of the Schrödinger-Madelung fluid is

$$
\mathbf{J}_{\mathrm{SchM}}=-\mathbf{v} \cdot \mathbf{P}^{\mathrm{r}}-\frac{\nu}{8}(\nabla \rho \nabla \cdot \mathbf{v}+\nabla \rho \cdot \nabla \mathbf{v}) .
$$

5.2.1. Remark. The results of this subsection are strange with the traditional interpretation of objectivity and frame independence. That question will be discussed from a more general point of view in the next section. 
5.2.2. Discussion. An important property of the Schrödinger-Madelung fluid is that if the motion of the fluid is vorticity free, $\nabla \times \mathbf{v}=\mathbf{0}$, then the mass and momentum balances can be transformed into and united in the Schrödinger equation. Hence the balance of momentum (56) can be derived from a Bernoulli equation (in a given inertial reference frame). Defining a scalar valued phase (velocity potential) by

$$
\mathbf{v}=\frac{\hbar}{m} \nabla S
$$

we obtain the Bernoulli equation observing that the second part of (81) is the gradient of

$$
\frac{\hbar}{m} \frac{\partial S}{\partial t}+\frac{\mathbf{v}^{2}}{2}-U_{\mathrm{SchM}}=0
$$

Then, introducing a single complex valued function $\psi:=R e^{i S}$ that unifies $R=$ $\sqrt{\rho}$ and $S$, it is easy to find that the sum of (55) multiplied by $i \hbar e^{i S} /(2 R)$ and (86) multiplied by $m R e^{i S}$ form together the Schrödinger equation for free particles:

$$
i \hbar \frac{\partial \psi}{\partial t}=-\frac{\hbar^{2}}{2 m} \Delta \psi
$$

It is remarkable that the structure of quantum mechanics appears in a classical thermodynamic approach without any explicit distinctive assumption related to the microscopic quantum world. In this sense the basic assumptions of our derivation are rather weak and very general.

\section{Summary AND OUTLOOK}

In this paper we have shown that the Second Law of thermodynamics provides a general, uniform, rigorous and constructive method to investigate weakly nonlocal extensions of classical and nonclassical non-equilibrium thermodynamics. We have seen how one can generate evolution equations for internal variables, to understand the constitutive structure of Classical Irreversible Thermodynamics and to restrict the pressure tensor of ideal and viscous Korteweg fluids.

In the suggested approach the choice of the constitutive state space is a physical question. The number of the necessary constraints for a given constitutive state space was introduced intuitively, according to our calculational experience, always looking forward to an explicit solution of the Liu equations. Cimmelli in 82 introduces a different philosophy, requiring that the number of the constraints must equal the number of constitutive state variables. Additional gradients of the constraints are to be introduced in case of necessity. In general, the method given in 37. introduces less additional constraints, therefore the results are more easily applicable. On the other hand, the method proposed in 82 is more cumbersome and the interpretation of the consequences of the Second Law can be less straightforward. However, it is based on a precise rule.

One of the interesting observations was that we were able to derive some restrictions to the reversible part of the evolution equations. There we have encountered equations of Euler-Lagrange type in case of zero entropy production in the corresponding interaction (static Ginzburg-Landau, dynamic degrees of freedom, Bohm-potential for Korteweg pressure). In a sense we were able to derive Hamiltonian variational principles, we have proved their existence from the Second Law of thermodynamics.

There are several other classical and nonclassical weakly nonlocal equations of physics that could be investigated in this general frame. We have analyzed weakly nonlocal extensions of the heat conduction equations (both Fourier and CattaneoVernotte) were researched, too. In this respect we have got that special kind of 
internal variables, the so called current multipliers can represent some kind of nonlocal effects in the theory. They can lead to theoretical structures like the GuyerKrumhansl equation of heat conduction [83. This concept is originated in general extensions of the entropy flux [84, 85]. These possibilities have been investigated together with higher order weakly nonlocal extensions in case of generalized heat conduction and generalized Ginzburg-Landau equations in [86, 50. The flux hierarchy of extended irreversible thermodynamics and the origin of balance form evolution equations for internal variables was researched in [87, 88].

On the other hand, we have researched phase separated multicomponent fluids and got a structure with natural instabilities (kind of nonlocal phase boundaries) similar to the Goodman-Cowin pressure [89, 90, 91.

However, why should we restrict ourselves to weakly nonlocal extensions of the constitutive functions? Why do not we consider time derivatives in exploiting the Second Law? The answer to this question leads to one of the most fundamental problems of physics, the question of objectivity, in particular, to the question of material frame indifference. In this paper we restricted ourselves to evolution equations and physical quantities given in an inertial reference frame. However, we know well, that the laws of physics are independent of an external observer. In particular, the constitutive functions can depend only on the material (e.g. on the motion of the continua) but not on the motion of an external observer. There are strong arguments that the usual formulation of the material frame indifference and the usual concept of objectivity is wrong $[92,93,94,95$. These investigations show well, that the core of the problem is in the usual intuitive concept of the nonrelativistic spacetime 96. Therefore, we need more rigorous analysis of the kinematics of classical continua from this point of view and an absolute, frame independent formulation of the exploitation method of the Second Law. As regards the new foundations of finite deformation kinematics the investigations of Fülöp seem to settle a good starting point 97 .

The results of those investigations are exploited in two key points in this paper. First of all, from the objectivity point of view, considering mathematical models of nonrelativistic spacetime, the successes of gradient extensions can be well understood. The gradient of a physical quantity is a spacelike component of a four-covector (spacetime quantity) and therefore it is independent of an observer 98: for inertial observers three-vectors are transforming by Galilei transformation, but three-covectors are invariant. Therefore gradients are objective. On the other hand, for fluids we have started our investigations with an explicitly velocity dependent constitutive state space. That is excluded by the old concept of objectivity, because the relative three-velocities are frame dependent. However, it is not excluded according to the new concept of objectivity, because the four-velocities are independent of the frame. Our treatment of a one component fluid here is not a true objective treatment, from more than one point of view. However, we have done the first steps toward the development of objective exploitation methods of the Second Law, too. As the problem of objectivity is in a sense more easily understandable from the point of view of a relativistic spacetime model, we have investigated the nonequilibrium thermodynamics of special relativistic fluids [99]. Moreover, we have started to investigate some possible practical consequences of incorporating our generalization of the objective time derivatives of rheology into a thermodynamic framework [100, 101.

Finally let us emphasize how surprising is our result regarding fluid mechanics. The original observation of Madelung was that the Schrödinger equation can be transformed into an interesting fluid mechanical form [102. The obervation of Bohm was that the same equation can be transformed into a Newtonian form [80]. 
In both cases the Schrödinger equation is postulated, coming out of the blue. Here we have derived the fluid mechanical equivalent of the Schrödinger equation in a very general framework, from a surprisingly minimal set of assumptions, from the basic balances, the Second Law of thermodynamics and nonlocality of the interactions were used. The existence of such derivation is a disturbing fact that is hardly understandable from the traditional point of view regarding the relation of the irreversible macro- and reversible microworld.

\section{ACKNOWLEDGEMENTS}

This research was supported by OTKA T048489. The author is grateful to T. Matolcsi and C. Papenfuss for the discussions and the corrections and for the valuable remarks of the referees.

\section{ApPEndix - FARKAS'S LEMma AND some of ITS CONSEQUENCES}

Lemma 7.1. (Farkas) Let $\mathbf{a}_{i} \neq \mathbf{0}$ be vectors in a finite dimensional vector space $\mathbb{V}, i=1 \ldots n$, and $S=\left\{\mathbf{p} \in \mathbb{V}^{*} \mid \mathbf{p} \cdot \mathbf{a}_{i} \geq 0, i=1 \ldots n\right\}$. The following statements are equivalent for all $\mathbf{b} \in \mathbb{V}$ :

(i) $\mathbf{p} \cdot \mathbf{b} \geq 0$, for all $\mathbf{p} \in S$.

(ii) There are nonnegative real numbers $\lambda_{1}, \ldots, \lambda_{n}$ such that $\mathbf{b}=\sum_{i=1}^{n} \lambda_{i} \mathbf{a}_{i}$.

Proof:

$(i i) \Rightarrow(i) \mathbf{p} \cdot \sum_{i=1}^{n} \lambda_{i} \mathbf{a}_{i}=\sum_{i=1}^{n} \lambda_{i} \mathbf{p} \cdot \mathbf{a}_{i} \geq 0$ if $\mathbf{p} \in S$.

$(i) \Rightarrow$ (ii) Let us consider a maximal, linearly independent subset $\mathbf{a}_{1}, \ldots, \mathbf{a}_{l}$ of $S$. Let $S_{0}=\left\{\mathbf{y} \in \mathbb{V}^{*} \mid \mathbf{y} \cdot \mathbf{a}_{i}=0, i=1 \ldots l\right\}$. Clearly $\emptyset \neq S_{0} \subset S$.

If $\mathbf{y} \in S_{0}$ then $-\mathbf{y}$ is also in $S_{0}$, therefore $\mathbf{y} \cdot \mathbf{b} \geq 0$ and $-\mathbf{y} \cdot \mathbf{b} \geq 0$ together. Therefore for all $\mathbf{y} \in S_{0}$ it is true that $\mathbf{y} \cdot \mathbf{b}=0$. As a consequence $\mathbf{b}$ is in the linear subspace generated by $\left\{\mathbf{a}_{i}\right\}$, that is there are real numbers $\lambda_{1}, \ldots, \lambda_{l}$ such that $\mathbf{b}=\sum_{i=1}^{l} \lambda_{i} \mathbf{a}_{i}$.

Moreover, for all $k \in\{1, \ldots, l\}$ there is a $\mathbf{p}_{k} \in \mathbb{V}^{*}$ such that $\mathbf{p}_{k} \cdot \mathbf{a}_{k}=1$ and $\mathbf{p}_{k} \cdot \mathbf{a}_{i}=0$ if $i \neq k$. Evidently, $\mathbf{p}_{k} \in S$ for all $k$, therefore $0 \leq \mathbf{p}_{k} \cdot \mathbf{b}=\mathbf{p}_{k} \cdot \sum_{i=1}^{l} \lambda_{i} \mathbf{a}_{i}=$ $\sum_{i=1}^{l} \lambda_{i} \mathbf{p}_{k} \cdot \mathbf{a}_{i}=\lambda_{k}$ is valid for all $k$. Lastly, we can choose zero multipliers for the vectors that are not independent.

Remark 7.1. In the following the elements of $\mathbb{V}^{*}$ are called independent variables and $\mathbb{V}^{*}$ itself is called the space of independent variables. The inequality in the first statement of the lemma is called objective inequality and the nonnegative numbers in the second statement are called Lagrange-Farkas multipliers. The inequalities determining $S$ are the constraints.

In the calculations an excellent reminder is to use Lagrange- Farkas multipliers similarly to the Lagrange multipliers in case of conditional extremum problems:

$$
\mathbf{p} \cdot \mathbf{b}-\sum_{i=1}^{n} \lambda_{i} \mathbf{p} \cdot \mathbf{a}_{i}=\mathbf{p} \cdot\left(\mathbf{b}-\sum_{i=1}^{n} \lambda_{i} \cdot \mathbf{a}_{i}\right) \geq 0, \quad \forall \mathbf{p} \in \mathbb{V}^{*}
$$

From this form we can read out the second statement of the lemma.

Remark 7.2. The geometric interpretation of the theorem is important and graphic: if the vector $\mathbf{b}$ does not belong to the cone generated by the vectors $\mathbf{a}_{i}$, there exists a hyperplane separating $\mathbf{b}$ from the cone.

7.1. Affine Farkas's lemma. This generalization of the previous lemma was first published simultaneously by A. Haar and J. Farkas as subsequent papers in the same journal, with different proofs [103, 104]. Later it was rediscovered independently by others several times (e.g. [105, 106]). 
Theorem 7.2. (Affine Farkas) Let $\mathbf{a}_{i} \neq \mathbf{0}$ be vectors in a finite dimensional vector space $\mathbb{V}$ and $\alpha_{i}$ real numbers, $i=1 \ldots n$ and $S_{A}=\left\{\mathbf{p} \in \mathbb{V}^{*} \mid \mathbf{p} \cdot \mathbf{a}_{i} \geq \alpha_{i}, i=1 \ldots n\right\}$. The following statements are equivalent for $a \mathbf{b} \in \mathbb{V}$ and a real number $\beta$ :

(i) $\mathbf{p} \cdot \mathbf{b} \geq \beta$, for all $\mathbf{p} \in S_{A}$.

(ii) There are nonnegative real numbers $\lambda_{1}, \ldots, \lambda_{n}$ such that $\mathbf{b}=\sum_{i=1}^{n} \lambda_{i} \mathbf{a}_{i}$ and $\beta \leq \sum_{i=1}^{n} \lambda_{i} \alpha_{i}$.

Proof:

$(i i) \Rightarrow(i) \mathbf{p} \cdot \mathbf{b}=\mathbf{p} \cdot \sum_{i=1}^{n} \lambda_{i} \mathbf{a}_{i}=\sum_{i=1}^{n} \lambda_{i} \mathbf{p} \cdot \mathbf{a}_{i} \geq \sum_{i=1}^{n} \lambda_{i} \alpha_{i} \geq \beta$.

$(i) \Rightarrow(i i)$ First we will show indirectly that the first condition of Farkas's lemma is a consequence of the first condition here, that is if (i) is true then $\mathbf{p} \cdot \mathbf{b} \geq 0$, for all $\mathbf{p} \in S$.

Thus let us assume the contrary, hence there is $\mathbf{p}^{\prime} \in S$, for which $\mathbf{p}^{\prime} \cdot \mathbf{b}<0$. Take an arbitrary $\mathbf{p} \in S_{A}$, then $\mathbf{p}+k \mathbf{p}^{\prime} \in S_{A}$ for all real nonnegative numbers $k$. But now $\left(\mathbf{p}+k \mathbf{p}^{\prime}\right) \cdot \mathbf{b}=\mathbf{p} \cdot \mathbf{b}+k \mathbf{p}^{\prime} \cdot \mathbf{b}<\beta$, if $k>\frac{\mathbf{p} \cdot \mathbf{b}-\beta}{-\mathbf{p}^{\prime} \cdot \mathbf{b}}$. That is a contradiction.

Therefore, according to Farkas's lemma exist nonnegative Lagrange-Farkas multipliers $\lambda_{1}, \ldots, \lambda_{n}$ such that $\mathbf{b}=\sum_{i=1}^{n} \lambda_{i} \mathbf{a}_{i}$. Hence $\beta \leq i n f_{p \in S_{A}}\left\{\mathbf{p} \cdot \sum_{i=1}^{n} \lambda_{i} \mathbf{a}_{i}\right\}=$ inf $f_{p \in S_{A}}\left\{\sum_{i=1}^{m} \lambda_{i} \mathbf{p} \cdot \mathbf{a}_{i}\right\}=\sum_{i=1}^{n} \lambda_{i} \alpha_{i}$.

Remark 7.3. The multiplier form is a good reminder again

$(\mathbf{p} \cdot \mathbf{b}-\beta)-\sum_{i=1}^{m} \lambda_{i}\left(\mathbf{p} \cdot \mathbf{a}_{i}-\alpha_{i}\right)=\mathbf{p} \cdot\left(\mathbf{b}-\sum_{i=1}^{m} \lambda_{i} \cdot \mathbf{a}_{i}\right)-\beta+\sum_{i=1}^{m} \lambda_{i} \alpha_{i} \geq 0, \quad \forall \mathbf{p} \in \mathbb{V}^{*}$.

Remark 7.4. The geometric interpretation is similar to the previous one, but with affine objects. If the line (one dimensional affine hyperplane) $(\mathbf{b}, \beta)$ does not belong to the (affine) cone generated by $\left(\mathbf{a}_{i}, \alpha_{i}\right)$ there exists an affine hyperplane separating b from the cone.

7.2. Liu's theorem. Here the constraints are equalities instead of inequalities, therefore the multipliers are not necessarily positive.

Theorem 7.3. (Liu) Let $\mathbf{a}_{i} \neq \mathbf{0}$ be vectors in a finite dimensional vector space $\mathbb{V}$ and $\alpha_{i}$ real numbers, $i=1 \ldots n$ and $S_{L}=\left\{\mathbf{p} \in \mathbb{V}^{*} \mid \mathbf{p} \cdot \mathbf{a}_{i}=\alpha_{i}, i=1 \ldots n\right\}$. The following statements are equivalent for $a \mathbf{b} \in \mathbb{V}$ and a real number $\beta$ :

(i) $\mathbf{p} \cdot \mathbf{b} \geq \beta$, for all $\mathbf{p} \in S_{L}$,

(ii) There are real numbers $\lambda_{1}, \ldots, \lambda_{n}$ such that

$$
\mathbf{b}=\sum_{i=1}^{n} \lambda_{i} \mathbf{a}_{i}
$$

and

$$
\beta \leq \sum_{i=1}^{n} \lambda_{i} \alpha_{i}
$$

Proof:

A straightforward consequence of the previous affine form of Farkas's lemma because $S_{L}$ can be given in a form $S_{A}$ with the vectors $\mathbf{a}_{i}$ and $-\mathbf{a}_{i}, i=1, \ldots, n$ : $S_{L}=\left\{\mathbf{p} \in \mathbb{V}^{*} \mid \mathbf{p} \cdot \mathbf{a}_{i} \geq \alpha_{i}, \mathbf{p} \cdot\left(-\mathbf{a}_{i}\right) \geq \alpha_{i}, i=1 \ldots n\right\}$.

Therefore there are nonnegative real numbers $\lambda_{1}^{+}, \ldots, \lambda_{n}^{+}$and $\lambda_{1}^{-}, \ldots, \lambda_{n}^{-}$such, that $\mathbf{b}=\sum_{i=1}^{n}\left(\lambda_{i}^{+} \mathbf{a}_{i}-\lambda_{i}^{-} \mathbf{a}_{i}\right)=\sum_{i=1}^{n}\left(\lambda_{i}^{+}-\lambda_{i}^{-}\right) \mathbf{a}_{i}=\sum_{i=1}^{n} \lambda_{i} \mathbf{a}_{i}$ and $\beta \leq \sum_{i=1}^{n}\left(\lambda_{i}^{+} \alpha_{i}-\right.$ $\left.\lambda_{i}^{-} \alpha_{i}\right)$.

Remark 7.5. The multiplier form is a help in the applications again

$0 \leq(\mathbf{p} \cdot \mathbf{b}-\beta)-\sum_{i=1}^{n} \lambda_{i}\left(\mathbf{p} \cdot \mathbf{a}_{i}-\alpha_{i}\right)=\mathbf{p} \cdot\left(\mathbf{b}-\sum_{i=1}^{n} \lambda_{i} \cdot \mathbf{a}_{i}\right)-\beta+\sum_{i=1}^{n} \lambda_{i} \alpha_{i}, \quad \forall \mathbf{p} \in \mathbb{V}^{*}$. 
Remark 7.6. In the theorem with Lagrange multipliers for local conditional extremum of differentiable function we apply exactly the above theorem of linear algebra after a linearization of the corresponding functions at the extremum point.

Considering the requirements of the applications we generalize Liu's theorem to take into account vectorial constraints:

Theorem 7.4. (vector Liu) Let $\mathbf{A} \neq \mathbf{0}$ in a tensor product $\mathbb{V} \otimes \mathbb{U}$ of finite dimensional vector spaces $\mathbb{V}$ and $\mathbb{U}$. Let $\alpha \in \mathbb{U}$ and $S_{L}=\left\{\mathbf{p} \in \mathbb{V}^{*} \mid \mathbf{p} \cdot \mathbf{A}=\alpha\right\}$. The following statements are equivalent for $a \mathbf{b} \in \mathbb{V}$ and a real number $\beta$ :

(i) $\mathbf{p} \cdot \mathbf{b} \geq \beta$, for all $\mathbf{p} \in S_{L}$.

(ii) There is a vector $\lambda$ in the dual of $\mathbb{U}$ such that

$$
\mathbf{b}=\mathbf{A} \cdot \lambda
$$

and

$$
\beta \leq \lambda \cdot \alpha
$$

Proof:

Let us observe that we can get back the previous form of the theorem by introducing a linear bijection $\mathbf{K}: \mathbb{U} \rightarrow \mathbb{R}^{n}$, a coordinatization in $\mathbb{U}$. Then by the vectors $\mathbf{a}_{i}:=\mathbf{A} \cdot \mathbf{K}^{*} \cdot \mathbf{e}_{i}$, where $\mathbf{e}_{i}$ is the standard $i$-th unit vector in $\mathbb{R}^{n}$, and the numbers $\alpha_{i}:=\mathbf{K} \cdot \alpha$, we obtain that $\mathbf{b}=\sum_{i=1}^{n} \lambda_{i} \mathbf{a}_{i}=\mathbf{A} \sum_{i=1}^{n} \lambda_{i} \mathbf{K}^{*} \mathbf{e}_{i}=\mathbf{A} \lambda$ where $\lambda:=\sum_{i=1}^{n} \lambda_{i} \mathbf{K}^{*} \mathbf{e}_{i}$

The previously excluded degenerate case of $\mathbf{A}=\mathbf{0}$ deserves a special attention. Then, of course, $\alpha=0$ and $S_{L}=\mathbb{V}^{*}$; this is, in fact, a degenerate case of all the previous theorems and evidently the following statements are equivalent for a $\mathbf{b} \in \mathbb{V}$ and a real number $\beta$ :

(i) $\mathbf{p} \cdot \mathbf{b} \geq \beta$ for all $\mathbf{p} \in \mathbb{V}^{*}$,

(ii) $\mathbf{b}=\mathbf{0}$ and $\beta \leq 0$.

In this case the assignment of the vector space of $\mathbb{V}^{*}$ is based on the inequality (i). We encountered various degeneracies in the calculations of the previous sections.

Remark 7.7. In continuum physics and thermodynamics the above algebraic theorems are applied to differential equations and inequalities. There the constraints are differential equations and therefore $\mathbb{V}$ is generated by derivatives of some constitutive functions in the differential equations. $\mathbb{V}^{*}$ is spanned by the process directions, the derivatives of the fields in the constitutive state space that are not already there. The corresponding form of (90) and (91) are called Liu equation(s) and the dissipation inequality, respectively.

\section{REFERENCES}

[1] J. D. Van der Waals. Thermodynamische theorie der kapillaritt unter voraussetzung stetiger dichtenderung. Zeitschrift für Physikalische Chemie, 13:657-725, 1894.

[2] D. Bedeaux, E. Johannessen, and A. Rojorde. A nonequilibrium van der Waals square gradient model. (I). the model and its numerical solution. Physica A, 330:329-353, 2003.

[3] E. Johannessen and D. Bedeaux. A nonequilibrium van der Waals square gradient model. (II). local equilibrium of the Gibbs surface. Physica A, 330:354-372, 2003.

[4] E. Johannessen and D. Bedeaux. A nonequilibrium van der Waals square gradient model. (III). heat and mass transfer coefficients. Physica A, 336:252-270, 2004.

[5] S. Kjelstup and D. Bedeaux. Non-equilibrium thermodynamics of heterogeneous systems. World Scientific, BerlinNew Jersey, etc., 2008.

[6] M. Grmela. Weakly nonlocal hydrodynamics. Physical Review E, 47(1):351-365, 1993.

[7] G. Lebon and M. Grmela. Weakly nonlocal heat conduction in rigid solids. Physics Letters A, 214:184-188, 1996.

[8] P. M. Mariano. Multifield theories in mechanics of solids. Advances in Applied Mechanics, 38:1-94, 2002. 
[9] G. A. Maugin. On the thermomechanics of continuous media with diffusion and/or weak nonlocality. Archive of Applied Mechanics, 75:723-738, 2006.

[10] V. A. Cimmelli and K. Frischmuth. Gradient generalization to the extended thermodynamic approach and diffusive-hyperbolic heat conduction. Physica B - Condensed matter, 400(12):257-265, 2007.

[11] A. Morro. A phase-field approach to non-isothermal transitions. Mathematical and computer modelling, 48(3-4):621-633, 2008.

[12] P. C. Hohenberg and B. I. Halperin. Theory of dynamic critical phenomena. Reviews of Modern Physics, 49(3):435-479, 1977.

[13] O. Penrose and P. C. Fife. Thermodynamically consistent models of phase-field type for the kinetics of phase transitions. Physica D, 43:44-62, 1990.

[14] M. Brokate and J. Sprekels. Hysteresis and Phase transitions, volume 121 of Applied Mathematical Sciences. Springer, New York, etc., 1996.

[15] I. S. Aranson and L. Kramer. The world of the complex Ginzburg-Landau equation. Reviews of Modern Physics, 74(1):99-143, 2002.

[16] G. A. Maugin. Nonlocal theories or gradient-type theories: a matter of convenience? Archives of Mechanics (Stosowanej), 31(1):15-26, 1979.

[17] G. A. Maugin. The principle of virtual power in continuum mechanics. Application to coupled fields. Acta Mechanica, 35:1-70, 1980.

[18] G. A. Maugin. Internal variables and dissipative structures. Journal of Non-Equilibrium Thermodynamics, 15:173-192, 1990.

[19] I. Vardoulakis and E. C. Aifantis. On the role of microstructure in behaviour of solids: effects of higher order gradients and internal inertia. Mechanics of Materials, 18:151-158, 1994.

[20] W. Kosiński and W. Wojno. A gradient generalization to internal state variable approach. Archive of Mechanics, 47(3):523-536, 1995.

[21] W. Kosiński. A modified hyperbolic framwork for thermoelastic materials with damage. In W. Kosiński, R. de Boer, and D. Gross, editors, Problems of Environmental and Damage Mechanics, pages 157-172, Warszawa, 1997. IPPT-PAN. Proceedings of SolMech'96 Conference, Mierki, Sept. 9-14. 1996, Poland.

[22] K. C. Valanis. A gradient theory of internal variables. Acta Mechanica, 116:1-14, 1996

[23] V. A. Cimmelli and W. Kosiński. Gradient generalization to internal state variables and a theory of superfluidity. Journal of Theoretical and Applied Mechanics, 35(4):763-779, 1997.

[24] K. C. Valanis. A gradient thermodynamic theory of self-organization. Acta Mechanica, 127:1-23, 1998 .

[25] P. B. Béda. Dynamic systems, rate and gradient effects in material instability. International Journal of Mechanical Sciences, 42:2101-2114, 2000.

[26] P. Ireman and Nguyen Q.-S. Using the gradients of temperature and internal parameters in Continuum Thermodynamics. Comptes Rendus Mecanique, 332:249-255, 2004.

[27] C. Papenfuss and S. Forest. Thermodynamical frameworks for higher grade material theories with internal variables or additional degrees of freedom. Journal of Non-Equilibrium Thermodynamics, 31(4):319-353, 2006.

[28] L. D. Landau and I. M. Khalatnikov. Ob anomal'nom pogloshehenii zvuka vblizi tochek fazovo perekhoda vtorovo roda. Dokladu Akademii Nauk, SSSR, 96:469, 1954. English translation: On the anomalous absorption of sound near a second order transition point. in: Collected papers of L. D. Landau, ed. D. ter Haar,(Pergamon, Oxford, 1965), pp. 626-633.

[29] P. Ván and W. Muschik. The structure of variational principles in nonequilibrium thermodynamics. Physical Review E, 52(4):3584-3590, 1995.

[30] M. Grmela and H. C. Öttinger. Dynamics and thermodynamics of complex fluids. I. Development of a general formalism. Physical Review E, 56(6):6620-6632, 1997.

[31] H. C. Öttinger and M. Grmela. Dynamics and thermodynamics of complex fluids. II. Illustrations of a general formalism. Physical Review E, 56(6):6633-6655, 1997.

[32] H. C. Öttinger. Beyond equilibrium thermodynamics. Wiley-Interscience, 2005.

[33] M. G. Gurtin. Generalized Ginzburg-Landau and Cahn-Hilliard equations based on a microforce balance. Physica D, 92:178-192, 1996.

[34] G. Capriz and P. M. Mariano. Multifield theories: and introduction. International Journal of Solids and Structures, 38:939-941, 2001. Preface to a special issue dedicated to multifield theories.

[35] P. Ván. Weakly nonlocal irreversible thermodynamics - the Guyer-Krumhansl and the CahnHilliard equations. Physics Letters A, 290(1-2):88-92, 2001. (cond-mat/0106568).

[36] P. Ván. Weakly nonlocal irreversible thermodynamics. Annalen der Physik (Leipzig), 12(3):146-173, 2003. (cond-mat/0112214).

[37] P. Ván. Exploiting the Second Law in weakly nonlocal continuum physics. Periodica Polytechnica, Ser. Mechanical Engineering, 49(1):79-94, 2005. (cond-mat/0210402/ver3). 
[38] K. Hutter. The foundations of thermodynamics, its basic postulates and implications. A review of modern thermodynamics. Acta Mechanica, 27:1-54, 1977.

[39] W. Muschik and H. Ehrentraut. An amendment to the Second Law. Journal of NonEquilibrium Thermodynamics, 21:175-192, 1996.

[40] Carl Eckart. The thermodynamics of irreversible processes, III. Relativistic theory of the simple fluid. Physical Review, 58:919-924, 1940.

[41] S. R. de Groot and P. Mazur. Non-equilibrium Thermodynamics. North-Holland Publishing Company, Amsterdam, 1962.

[42] B. D. Coleman and V. J. Mizel. Existence of caloric equation of state in thermodynamics. Journal of Chemical Physics, 40:1116-1125, 1964

[43] I-Shih Liu. Method of Lagrange multipliers for exploitation of the entropy principle. Archive of Rational Mechanics and Analysis, 46:131-148, 1972.

[44] V. Triani, C. Papenfuss, V. A. Cimmelli, and W. Muschik. Exploitation of the Second Law: Coleman-Noll and Liu procedure in comparison. Journal of Non-Equilibrium Thermodynamics, 33:47-60, 2008.

[45] I. Müller. On the frame dependence of stress and heat flux. Archive of Rational Mechanics and Analysis, 45:241-250, 1972.

[46] W. Muschik, C. Papenfuss, and H. Ehrentraut. A sketch of continuum thermodynamics. Journal of Non-Newtonian Fluid Mechanics, 96:255-290, 2001.

[47] R. A. Hauser and N. P. Kirchner. A historical note on the entropy principle of Müller and Liu. Continuum Mechanics and Thermodynamics, 14:223-226, 2002. Lecture held on CIMRF'01, Berlin, 3-6 September, 2001.

[48] H. Minkowski. Geometrie der Zahlen. Teubner, Leipzig und Berlin, 1896.

[49] Gy. Farkas. A Fourier-féle mechanikai elv alkalmazásai. Mathematikai és Természettudományi Értesítö, 12:457-472, 1894. in Hungarian.

[50] P. Ván. The Ginzburg-Landau equation as a consequence of the Second Law. Continuum Mechanics and Thermodynamics, 17(2):165-169, 2005. (reduced cond-mat/0210402).

[51] P. Ván, A. Berezovski, and Engelbrecht J. Internal variables and dynamic degrees of freedom. Journal of Non-Equilibrium Thermodynamics, 33(3):235-254, 2008. cond-mat/0612491.

[52] G. A. Maugin and W. Muschik. Thermodynamics with internal variables. Part I. General concepts. Journal of Non-Equilibrium Thermodynamics, 19:217-249, 1994.

[53] B. D. Coleman and M. E. Gurtin. Thermodynamics with internal state variables. The Journal of Chemical Physics, 47(2):597-613, 1967.

[54] W. Muschik. Internal variables in non-equilibrium thermodynamics. Journal of NonEquilibrium Thermodynamics, 15:127-137, 1990.

[55] J. Verhás. Thermodynamics and Rheology. Akadémiai Kiadó and Kluwer Academic Publisher, Budapest, 1997.

[56] G. A. Maugin. Internal variables and dissipative structures. Journal of Non-Equilibrium Thermodynamics, 15:173-192, 1990.

[57] G. Maugin. The thermomechanics of nonlinear irreversible behaviors (An introduction). World Scientific, Singapure-New Jersey-London-Hong Kong, 1999.

[58] Jüri Engelbrecht. Nonlinear wave mechanics. Kluwer Academic Publishers, DordrechtBoston-London, 1997.

[59] G. A. Maugin and W. Muschik. Thermodynamics with internal variables. Part II. Applications. Journal of Non-Equilibrium Thermodynamics, 19:250-289, 1994.

[60] L. Onsager. Reciprocal relations of irreversible processes I. Physics Reviews, 37:405-426, 1931.

[61] L. Onsager. Reciprocal relations of irreversible processes II. Physics Reviews, 38:2265-2279, 1931.

[62] L. Onsager and S. Machlup. Fluctuations and irreversible processes. Physical Review, 91(6):1505-1512, 1953.

[63] S. Machlup and L. Onsager. Fluctuations and irreversible processes. II. Systems with kinetic energy. Physical Review, 91(6):1512-1515, 1953.

[64] H. G. B. Casimir. On Onsager's principle of microscopic reversibility. Reviews of Modern Physics, 17:343-350, 1945.

[65] G. A. Maugin and R. Drouot. Internal variables and the thermodynamics of macromolecule solutions. International Journal of Engineering Science, 21(7):705-724, 1983.

[66] R. Drouot and G. A. Maugin. Application of diffusive internal variables in complex fluids. Journal of Non-Newtonian Mechanics, 96:31-43, 2001.

[67] J. Engelbrecht and M. Vendelin. Microstructure described by hierarchical internal variables. Rendiconti del Seminario Matematico, Universitá e Politecnico di Torino, 58(1):93-98, 2000. 
[68] J. Kestin. Internal variables in the local-equilibrium approximation. Journal of NonEquilibrium Thermodynamics, 18:360-379, 1993.

[69] J. Verhás. An application of Gyarmati's wave approach. Acta Physica Hungarica, 66(14):95-97, 1989.

[70] J. Verhás. Non-equilibrium thermodynamics and the dynamics of particles. In O. Gues G. Iooss and A. Nouri, editors, Trends in Applications of Mathematics to Mechanics, pages 138-146, Boca Raton-London-New York-Washington, D. C., 2000. Chapman and Hall/CRC.

[71] I. Gyarmati. Non-equilibrium Thermodynamics /Field Theory and Variational Principles/. Springer Verlag, Berlin, 1970.

[72] P. Glansdorff and I. Prigogine. Thermodynamic Theory of Structure, Stability and Fluctuations. Wiley-Interscience, London-etc., 1971.

[73] F. Márkus and K. Gambár. A variational principle in thermodynamics. Journal of NonEquilibrium Thermodynamics, 16(1):27-31, 1991.

[74] P. Ván and B. Nyíri. Hamilton formalism and variational principle construction. Annalen der Physik (Leipzig), 8:331-354, 1999.

[75] I. Müller. A thermodynamic theory of mixtures of fluids. Archive of Rational Mechanics and Analysis, 28:1-39, 1968.

[76] P. Ván and T. Fülöp. Weakly nonlocal fluid mechanics - the Schrödinger equation. Proceedings of the Royal Society, London A, 462(2066):541-557, 2006. (quant-ph/0304062).

[77] P. Ván and T. Fülöp. Stability of stationary solutions of the Schrödinger-Langevin equation. Physics Letters A, 323(5-6):374-381, 2004. (quant-ph/0304190).

[78] J. E. Dunn and J. Serrin. On the thermomechanics of interstitial working. Archive of Rational Mechanics and Analysis, 88:95-133, 1985.

[79] N. P. Kirchner and K. Hutter. Elasto-plastic behaviour of a granular material with and additional scalar degree of freedom. In W. Ehlers and J. Blum, editors, Porous Media: Theory, Experiments and Numerical Applications, pages 147-168. Springer, 2002.

[80] D. Bohm. Quantum Theory. Prentice-Hall, New York, 1951.

[81] P. R. Holland. The Quantum Theory of Motion. Cambridge University Press, Cambridge, 1993.

[82] V. A. Cimmelli. An extension of Liu procedure in weakly nonlocal thermodynamics. Journal of Mathematical Physics, 48:113510, 2007.

[83] P. Ván. Internal thermodynamic variables and the failure of microcracked materials. Journal of Non-Equilibrium Thermodynamics, 26(2):167-189, 2001.

[84] J. Verhás. On the entropy current. Journal of Non-Equilibrium Thermodynamics, 8:201-206, 1983.

[85] B. Nyíri. On the entropy current. Journal of Non-Equilibrium Thermodynamics, 16:179-186, 1991.

[86] V. Ciancio, V. A. Cimmelli, and P. Ván. On the evolution of higher order fluxes in nonequilibrium thermodynamics. Mathematical and Computer Modelling, 45:126-136, 2007. cond-mat/0407530

[87] V. A. Cimmelli and P. Ván. The effects of nonlocality on the evolution of higher order fluxes in non-equilibrium thermodynamics. Journal of Mathematical Physics, 46(11):112901-15, 2005. cond-mat/0409254

[88] V. A. Cimmelli and P. Ván. First order weak nonlocality in extended thermodynamics of rigid heat conductors. In R. Monaco, G. Mulone, S. Rionero, and T. Ruggeri, editors, Proceedings of WASCOM'05, pages 122-127, New Jersey-London-etc., 2006. World Scientific. 13th Conference on Waves and Stability in Continuous Media, 2005 June 19-25, Catania.

[89] M. A. Goodman and S. C. Cowin. A continuum theory for granular materials. Archive of Rational Mechanics and Analysis, 44:249-266, 1972.

[90] P. Ván. Weakly nonlocal continuum theories of granular media: restrictions from the Second Law. International Journal of Solids and Structures, 41(21):5921-5927, 2004. (cond-mat/0310520).

[91] P. Ván. One and two component fluids: restrictions from the Second Law. Physica A, 340(13):418-426, 2004.

[92] T. Matolcsi and T. Gruber. Spacetime without reference frames: An application to the kinetic theory. International Journal of Theoretical Physics, 35(7):1523-1539, 1996.

[93] T. Matolcsi and P. Ván. Can material time derivative be objective? Physics Letters A, 353:109-112, 2006. math-ph/0510037.

[94] T. Matolcsi and P. Ván. On the objectivity of time derivatives. Atti dell'Accademia Peloritana dei Pericolanti, Classe di Scienze Fisiche, Matematiche e Naturali, Suppl. I., 86:1-13, 2008.

[95] M. Frewer. More clarity on the concept of material frame-indifference on classical continuum mechanics. Acta Mechanica, 202:213-246, 2009. 
[96] T. Matolcsi and P. Ván. Absolute time derivatives. Journal of Mathematical Physics, 48:053507-19, 2007. math-ph/0608065

[97] T. Fülöp. A new interpretation of the kinematics of continua. In T. Fülöp, editor, New results in continuum physics, volume 8 of Notes on Engineering Geology and Rock Mechanics, chapter 3, pages 55-99. BME Publisher, Budapest, 2008. in Hungarian.

[98] T. Matolcsi. Spacetime Without Reference Frames. Akadémiai Kiadó Publishing House of the Hungarian Academy of Sciences), Budapest, 1993.

[99] P. Ván. Internal energy in dissipative relativistic fluids. Journal of Mechanics of Materials and Structures, 3(6):1161-1169, 2008. Lecture held at TRECOP'07, arXiv:07121437 [nuclth].

[100] Ván P. and Asszonyi Cs. General principles of continuum mechanics. In Cs. Asszonyi, editor, Constitutive functions of isotropic materials, volume 3 of Notes on Engineering Geology and Rock Mechanics, chapter 2, pages 25-87. Technical University Press, Budapest, 2006. in Hungarian.

[101] P. Ván. Objective time derivatives in non-equilibrium thermodynamics. Proceedings of Estonian Academy of Sciences, 57(3):127, 2008.

[102] E. Madelung. Quantentheorie in hydrodynamischer Form. Zeitschrift für Physik, 40:322326, 1926. in German.

[103] A. Haar. A lineáris egyenlőtlenségekről. Mathematikai és Természettudományi Értesítő, 36:279-296, 1918. in Hungarian.

[104] Gy. Farkas. Egyenlőtlenség alkalmazásának új módjai. Mathematikai és Természettudományi Értesítö, 36:297-308, 1918. in Hungarian.

[105] J. von Neuman. Discussion of a maximum principle. In A. H. Taub, editor, Collected Works, volume VI, pages 89-95, New York and Oxford, 1961. Pergamon Press.

[106] A. Schriver. Theory of linear and integer programming. John Wiley and Sons, Chicesteretc.., 1998.

Department of Theoretical Physics, KFKi, Research Institute of Particle and Nuclear Physics,, Konkoly Thege Miklós út 29-33., 1525 Budapest, Hungary, Department of Energy Engineering, Budapest University of Technology and Economics, Bertalan Lajos u. 4-6. 1111 Budapest, Hungary

E-mail address: vpet@rmki.kfki.hu 